Cult Med Psychiatry. Author manuscript; available in PMC 2016 December 16.

Published in final edited form as:

Cult Med Psychiatry. 2010 March ; 34(1): 56-105. doi:10.1007/s11013-009-9161-3.

\section{Therapeutic Processes and Perceived Helpfulness of Dang-Ki (Chinese Shamanism) from the Symbolic Healing Perspective}

\author{
Boon-Ooi Lee, \\ Singapore 637616, Singapore \\ Laurence J. Kirmayer, and \\ McGill University, 845 Sherbrooke Street, West Montreal, QC H3A 2T5, Canada \\ Danielle Groleau \\ McGill University, 845 Sherbrooke Street, West Montreal, QC H3A 2T5, Canada
}

National Institute of Education, Nanyang Technological University, No. 1, Nanyang Walk,

\section{Abstract}

This study focuses on the therapeutic process and perceived helpfulness of dang-ki, a form of Chinese shamanistic healing, in Singapore. It aims to understand the healing symbols employed in dang-ki, whether or not patients find them helpful and whether their perceived helpfulness can be explained by the symbolic healing model (Dow, Am Anthropol 88(1):56-69, 1986; Levi-Strauss, Structural anthropology. Basic Books, New York, 1963). Although many researchers have applied this model to explain the efficacy of shamanistic healings, they did not directly provide empirical support. Furthermore, the therapeutic process of a shared clinical reality as proposed by the model may be achievable in small-scale traditional societies that are culturally more homogeneous than in contemporary societies that are culturally more diversified due to globalization and immigration. Patients may hold multidimensional health belief systems, as biomedicine and alternative healing systems coexist. Thus, it would be interesting to see the relevance and applicability of the symbolic healing model to shamanistic healing in contemporary societies. In this study, ethnographic interviews were conducted with 21 patients over three stages: immediately before and after the healing and approximately 1 month later. The dang-ki healing symbols were identified by observing the healing sessions with video recording. Results show that dang-kis normally applied more than one method to treat a given problem. These methods included words, talismans and physical manipulations. Overall, 11 patients perceived their consultations as helpful, 4 perceived their consultations as helpful but were unable to follow all recommendations, 5 were not sure of the outcome because they had yet to see any concrete results and only 1 patient considered his consultation unhelpful. Although the symbolic healing model provides a useful framework to understand perceived helpfulness, processes such as enactment of a common meaning system and symbolic transformation are complex and dynamic, and may be carried over several healing sessions.
\end{abstract}

\title{
Keywords
}

Symbolic healing; Chinese shamanistic healing; Spiritual medium; Dang-ki; Perceived helpfulness 


\section{Introduction}

The development of shamanism, the concept and origin of the word "shamanism" and the geographical distributions of shamanism are complex, controversial and unclear (Balzer 1996; Jakobsen 1999; Laufer 1917). This is partially because researchers have defined shamanism differently, according to their sites of research, theoretical disciplines and interests (D'Anglure 1997). The narrow definition of shamanism limits it to a healing system found among hunting peoples that centers on the use of magical or spiritual powers of diagnosis and intervention acquired with the aid of animal spirits (Vitebsky 2001). But much broader uses of the term have become popular, based on generalizing certain aspects of the shamanic complex. For example, psychologists and psychiatrists may focus on the use of trance or altered states of consciousness as a distinguishing feature of shamanism, whereas anthropologists may emphasize specific symbolic meanings and sociopolitical aspects. For the purpose of this study, a shaman is defined as a mediator between the human and the spiritual worlds (Balzer 1996; D'Anglure 1997). Shamanism is "a practice in which a person deliberately alters her consciousness for the purpose of interacting with spirits in order to serve the community in which she finds herself" (Baruss 2003, p. 136). The most common role of shamans in their service to the community is healing (Krippner 2002, 2004; Winkerlman 2000).

While shamanism can be found across times and cultures, it is an interesting phenomenon especially in contemporary societies because it has been utilized alongside psychotherapy and biomedicine for managing mental and physical health (Chong et al. 2005; Finkler 2004; Heinze 1997; Kirmayer 2004; Kleinman 1980: Nguyen 2008). This suggests that shamanism may serve certain therapeutic functions that are beyond the reach of modern health care systems. The persistence and popularity of shamanism may also imply that it may be effective in fulfilling its therapeutic functions. Understanding whether it is effective, and why and how it is effective (i.e., therapeutic processes and outcomes), may help enrich the theory and practice of psychotherapy and biomedicine. Thus, a number of psychotherapy researchers have advocated collaboration with shamans or incorporation of shamanistic healing concepts into psychotherapy (Costantino et al. 1986; Helms and Cook 1999; Kreisman 1975; Laird 1984; Parks 2003; Yeh et al. 2004).

In this study, we focus on dang-ki, which is a form of Chinese shamanistic healing in Singapore. Our aims are to find out whether it is helpful and, if so, whether its therapeutic process can be explained from a symbolic healing perspective (Dow 1986; Levi-Strauss 1963). We first describe the characteristics of dang-ki, followed by the theoretical framework of symbolic healing.

\section{Brief Description of Dang-ki}

The term dang-ki is made up of two Chinese characters, pronounced in Chinese Hokkien.

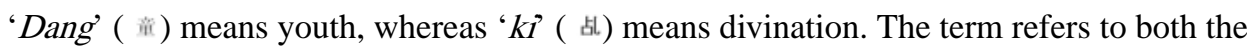
practice and the practitioner. The use of 'dang' may imply that dang-ki is a youth, but this is not often the case. Because dang-ki is most likely to be a male, the pronoun "he" is used 
throughout this article. A dang-ki is a person who enters a trance and is possessed by a helping deity offering aid to supplicants. It is a popular form of shamanistic healing among Hokkien, a main Chinese dialect group in Singapore and Southeast Asia. As health-related issues are the most common problem presented to dang-ki (DeBernardi 2006; Elliott 1955; Heinze 1997; Kleinman 1980), his main role is healing. In Singapore, dang-kis used to practice in temples or rural residential houses (Elliott 1955; Heinze 1997). But nowadays, due to urbanization, most of them practice from shrines located in private residences (Chan 2006) or high-rise public apartments.

Although many researchers have studied dang-ki (Ackerman 2001; Chan 2006; DeBernardi 2006; Elliott 1955; Fidler 1993; Holbrook 1975; B. O. Lee 2002; R. Lee 1986; Tseng 1978; Zhang 1996), only a few have attempted to examine its healing effectiveness (Kleinman 1980; Kleinman and Gale 1982; Kleinman and Sung 1979). Kleinman and his associates observed dang-ki healings, interviewed some patients before the healings and conducted follow-up interviews 2 months later with 12 patients. Ten patients reported at least partially effective treatment, six patients were regarded as completely cured and only two patients were listed as treatment failures. Thus, dang- $k i$ is generally considered effective. However, Kleinman's findings may not be generalized to the practice of dang-ki in contemporary Singapore because their study was conducted in Taiwan in the 1970s. Furthermore, they presented only the outcomes, without the detailed dynamic and processes of the dang-ki healing in relation to the self-reported efficacy. A focus on therapeutic dynamic and processes provides additional insight into healing outcome (see Kapferer 2004; Turner 1977). Hence, this study updates and contributes to the existing literature of dang-ki efficacy and shamanistic healing in general.

\section{Symbolic Healing Model}

If dang-ki "works," it may be, in part, because of the dang-ki's ability to symbolically transform patients' experiences within a culturally legitimized myth (see Frank and Frank 1991). Using the example of Cuna Indian shamans in Panama, Levi-Strauss (1963) discussed how the shamans used specific incantations to facilitate difficult childbirth. He argued that the shaman's song provided a symbolic account that metaphorically manipulated the sick organ. The healing was possible as the incantation was based on a clinical reality shared by both the shaman and the sick woman.

Building on Levi-Strauss's work, Dow (1986) proposed that all therapeutic systems, including shamanism and biomedicine, are based on healing symbols that healers use for transforming patients' experiences. Examples of these healing symbols are medications, chanting, ritual objects, ritual acts and surgery. They are "symbolic" because they are socioculturally and therapeutically meaningful to users. However, being symbolic does not imply the absence of active therapeutic ingredients. Healing symbols can have both symbolic (psychological) and instrumental (direct physical effect) value (Castillo 2001; Welch 2003; Winkerlman 2000). For example, although herbs and other medicinal products prescribed by shamans may have actual pharmacological ingredients (Winkerlman 2000; Zhang 1996), their effects are not solely physiological but are partially contingent on how meaningful they are to patients (see Moerman 2002). Patients may believe that the herbs are 
therapeutic because they are prescribed by a powerful god or shaman. In this sense, patients are not passive recipients but are active in responding to treatments (B. O. Lee 2006; Walach and Jonas 2004).

Every system of symbolic healing is based on a mythic world or a cultural experiential reality (Dow 1986). The mythic world is located differently in different traditions: some are located in a supernatural realm, as in the case of shamanism; others, in a scientific realm, as in the case of contemporary psychotherapy and biomedicine. The success of treatment depends on the healer's ability to enact a mythic world consistent with the patient's belief system or to persuade the patient that his or her condition can be defined in terms of the given mythic world. The healer attaches the patient's emotions to the transactional symbols particularized from the general myth and then manipulates the symbols to emotionally transform the patient. More specifically, patients' subjective states including emotions and bodily sensations become symbolically "objectivized" and are incorporated into pre-existing cultural patterns (Levi-Strauss 1963). Healing symbols provide "order" in the disordered situation such as disease (Peacock 1984). Their sensual aspects embody the physical meanings of disease, while their conceptual aspects classify the experience of disease. Accordingly, they transform confusion into order and meaning, private experience of disease into collective experience and nature into culture.

Similarly, Kleinman (1980) proposes that healing rituals move through three therapeutic stages: the sickness is labeled with an appropriate and sanctioned cultural category. The label is then ritually manipulated and transformed. Finally, a new label (e.g., cure, well) is applied and sanctioned as a meaningful symbolic form that may be independent of behavioral or social changes. Patients and their healers jointly construct clinical reality that functions as a "real world" for them. Any explanatory models (EMs) that have been taken from this reality are unlikely to be effective because they have lost their contextual meanings. For example, shamanistic healing is unlikely to be effective when it is conducted in a Western medical setting. Hence, Kleinman asserts that it is the clinical reality that is therapeutically effective, not the practitioners.

Many researchers have attempted to explain the efficacy of shamanistic healings from a symbolic healing perspective. Using two cases, Nguyen (2008) demonstrated how the interactions between the patient's and a shaman's EMs were related to healing outcomes in Vietnam. The shaman was able to heal a male patient by successfully labeling him with a culturally sanctioned category (possession) and then transforming his experiences through exorcism and negotiations. But the shaman failed to help a female patient because the two of them were oriented toward different religious systems (Mother Goddess vs. Buddha).

Nguyen's research is limited by focusing on the healing structure without providing sufficient process data to help understand the healing dynamic over time-a point that we discuss later.

Other researchers have also analyzed the shamanistic efficacy based on the symbolic healing model but did not directly provide empirical supports (Castillo 2001; Desjarlais 1989; Li 2003). For example, Desjarlais reported how a Nepali shaman had described metaphorical and real physical landscapes to his patients during his magical flight to search for lost souls. 
The researcher believed that the landscape was familiar to the patients because it was located within the vicinity of their village. During the magical flight, the shaman might have restructured the images of the landscape representing certain domains of the patients' experiences. The patients were guided and transformed experientially by moving from one terrain to another. However, it remains unknown whether the patients had really been transformed in this manner. They were not interviewed or assessed pre or post healing sessions, or about whether they understood the shaman's descriptions or, most importantly, about whether they found the healing helpful.

Likewise, whether the woman patients of Cuna Indian shamans, as reported by Levi-Strauss (1963), really found the incantations helpful is highly speculative. Sherzer (1983) argued that because the incantation was an esoteric language, the patients could not necessarily follow the specific process that Levi-Strauss described. Likewise, in Taiwan, many patients are not familiar with the symbolic meanings underlying some dang-ki rituals (Kleinman 1980). In Mexico, although patients do not understand why their shamans behave in certain ways during the healing trance, they accept the shamans' injunctions without question (Finkler 1980). In Japan, the Itako shamans speak in a dialect that is not comprehensible to their urban patients (Freeman 1998). More recently, Lindquist (2007) demonstrated how transformation occurred in a patient who had no knowledge of the language of the shamanistic healing in which he was involved in Moscow. If patients do not understand their shamans, why do they still find the consultation helpful? Do these examples suggest that a shared clinical reality is a necessary but not sufficient contributing factor to healing efficacy?

A mere focus on the form and structure (i.e., symbols) in healing may overlook its transformative performance, process and dynamic. Turner (1977, p. 77) regards ritual symbols as "dynamic systems of signifiers, signifieds, and changing modes of signification in temporal sociocultural processes." These symbols, which are the smallest units of analysis in any ritual healing, include objects, activity, relationship, word, gesture and spatial arrangement in a healing context (symbol vehicle). Their meanings are not fixed and may be interpreted differently by different people, who may in turn add their personal meanings to the public meanings of the symbols. For Obeyesekere (1985, 1990), personal meanings or symbols are psychodynamically rooted in unconscious. Deep motivations and intrapsychic conflicts are being expressed, objectified and transformed through pre-existing cultural symbols (embedded in rituals and performances) that are significant to the individuals and public. For example, in a case Obeyesekere (1990) described, the mother goddess (cultural symbol) is simultaneously an infantile identification with the informant's mother (personal symbol). In this sense, the personal and cultural symbols are part of the same symbolic form.

Building on Turner's (1977) work, Kapferer (2004, p. 50) argues that "although the representational, meaning-driven, symbolic perspective continues to be important, there is a shift to viewing ritual as a dynamic for the production of meaning rather than seeing it as necessarily predominantly meaningful in itself...." He conceives of ritual healing as a "virtuality" that is a dynamic process and a kind of phantasmagoric space involving the structuration of perception and cognition in which human potentials both of experience and of meaningful construction may be formed and transformed. Hence, meaning is not simply 
shared between patient and healer but is also the dynamic process whereby the patient is responding to and constructing his or her healing experience.

Taken together, Turner (1977), Obeyesekere $(1985,1990)$, and Kapferer (2004) have clearly broadened Dow's model by emphasizing the depth, dynamic and process of ritual healing. These are some of the areas we attempt to explore in the present study.

Another challenge that the symbolic healing model faces is that a shared clinical reality may be achievable in small-scale traditional societies that are culturally more homogeneous than in contemporary societies that are culturally more diversified due to globalization and immigration. In these societies, "suffers and healers may live in different local worlds and may not share the same notions of the roles of patient and healer, the appropriate place and time for healing, the meaning of symbolic acts, and the expected outcome" (Kirmayer 2003, p. 249; also see Lindquist 2007). Furthermore, patients may hold multidimensional health belief systems, as biomedicine and alternative healing systems coexist. Thus, it would be interesting to determine the relevance and applicability of symbolic healing model to shamanistic healing in contemporary societies.

\section{The Present Study}

Findings reported in this paper are based on a portion of data collected from an ongoing study on dang-ki healing in Singapore. Given the few studies on dang-ki healing, this study is the first step of research into its processes and outcomes in the 21st century. Singapore provides a unique sociocultural environment for such research because it is a metropolitan urban state where Asian and Western values interact, and traditional healing systems and biomedicine coexist. As the first step into the enquiry, this study adopts an explorative approach to understand how dang-kis' patients construct their healing experiences, particularly their perceived helpfulness.

Prior to conducting the formal field research, we had informal interactions with several shrine helpers and attendees. What emerged consistently from their sharing was the concern about "whether the god is able to help them solve their issues." This concern was especially salient among people presenting health-related problems. The notion of helpfulness appears to be the central evaluative criterion of the dang-ki healing among these patients. Dang-ki, similar to other shamanistic healings, is a performance-centered ritual where performance efficacy is an important indicator of the shaman's ability to solve practical problems (see Lindquist 2007) since most of the problems that patients present to dang-kis are practical by nature, namely, health-related problems (DeBernardi 2006; Elliot 1955; Heinze 1997).

Patients are concerned about shamans' helpfulness, as they often use shamanistic healing as a last resort after their illness condition has not been improved with biomedicine (Finkler 2004; Yeh et al. 2004). Some Chinese patients consult several dang-kis for intractable problems because of dissatisfaction with results or because they want second opinions (DeBernardi 2006).

Although the dang-ki's helpfulness is the main concern, its meanings and definitions are likely to vary across individuals (see Kirmayer 2004). In this study, we are interested in how 
patients construct and perceive the dang-ki's helpfulness rather than in objective outcomes derived from experimental methods such as randomized clinical trials that are unable to capture the rich and dynamic healing experiences and transformation evoked in ritual healing (see Waldram 2000). While objective outcome is derived from systematizing or scientific thinking, perceived helpfulness is a form of "everyday thinking" that is pragmatic and that does not aim to achieve consistency and coherence but, rather, concerns everyday activities and needs (Young 1976).

Based on the above discussion, three research questions were generated to guide this study:

1. What are the healing symbols employed in dang-ki healing?

2. From patients' perspectives, do they find dang-ki helpful in terms of these healing symbols? If so, why and how do they perceive dang-ki to be helpful (i.e., meanings of helpfulness)?

3. Can this perceived helpfulness be explained by shared clinical reality and patients' understanding of the purpose and contents of the healing symbols?

\section{Method}

\section{Setting and Research Participants}

Twenty-one patients (mean age $=44.29, \mathrm{SD}=11.83$; range $=26-67$ ) from three shrines took part in this study. They were all Chinese and most of them were female (62\%), were Taoist (38\%), had secondary-school qualification (33\%), were married (53\%) and had a monthly total household income of $\mathbf{S} \$ 2,000-\mathrm{S} \$ 3,999(48 \%) .{ }^{1}$ Table 1 presents their detailed demographic background.

During the interviews, 17 patients spoke Mandarin Chinese, whereas 4 spoke English. $^{2}$ Thirteen were regular attendees who had visited the shrine for more than one year (1-30 years), whereas eight were newcomers who had visited the shrine for less than a year (one to three visits).

\section{Recruitment of Shrines and Interviewees}

The three dang-ki shrines involved in this study were recruited through an informal Taoist organization in Singapore. To recruit interviewees, every individual waiting in the shrine lobby for the dang-ki consultation was approached. Potential interviewees had to be adults who were able to speak Mandarin Chinese or English, and who presented health or psychosocial issues. ${ }^{3}$ Twenty-two attendees (i.e., patients) agreed, signed the consent forms and completed the full interview. One of them (Case 7) was eliminated from subsequent data analysis because of irregular data. ${ }^{4}$

\footnotetext{
${ }_{1}^{1}$ One U.S. dollar is equivalent to 1.44 Singapore dollars (as of August 2009).

2 In Singapore, English is one of the four official languages, including Chinese, Malay and Tamil.

${ }^{3}$ Given that health-related issues are the most common issues presented to dang-kis (DeBernardi 2006; Kleinman 1980), and to enable a systematic comparison across patients, only those presenting psychosocial and health-related issues were interviewed.

${ }^{4}$ The issue that the female patient presented during the prehealing interview was not the same as the issue she subsequently presented to the dang-ki. She also refused to take part in the posthealing interview, without giving a reason.
} 


\section{Research Protocol}

Research was carried out in three stages. Stage 1 (the prehealing interview) elicited the patients' illness narratives including EMs and prehealing expectations. Stage 2 involved observation of the healing process with video recording, with attention to the types of healing symbols (e.g., talisman), and the interactions between patient and dang-ki. Stage 3 (the posthealing interview) explored patients' perceived helpfulness of the healing experiences immediately after consultation.

All three research stages were carried out at the shrines. Follow-up interviews were conducted with patients approximately one month later by telephone to evaluate the perceived effects. One patient (Case 16) was contactable only after 8 months. As observed by Kleinman (1980), when patients are asked to evaluate the treatments while still in the shrine, they may give a positive evaluation for fear of offending the deities, dang-ki or other cult members. Thus, a follow-up evaluation at a later stage and at other places is necessary to elicit further information.

\section{Interview Questionnaire}

The interview used the McGill Illness Narrative Interview (MINI; Groleau et al. 2006), which was adapted according to the three research questions. The modified MINI consisted of two parts: the prehealing interview and the posthealing interview.

- $\quad$ Research Question 1: What are the healing symbols employed in dang-ki healing? Information for this question was derived from the posthealing interview (e.g., "Did the god conduct any rituals and give you any medicines or recommendations to follow?") and from observation of the healing session.

- $\quad$ Research Question 2: From the patients' perspectives, do they find dang-ki helpful in terms of these healing symbols? If so, why and how do they perceive dang-ki to be helpful (i.e., meanings of helpfulness)?

This question was aimed at exploring the patients' perceived helpfulness of these healing symbols. Information for this question was derived from the posthealing interview (e.g., "Are the treatments, medicines or recommendations helpful, and why?").

- $\quad$ Research Question 3: Can this perceived helpfulness be explained by shared clinical reality and patients' understanding of the purpose and contents of the healing symbols?

Shared clinical reality was explored by comparing patients' EMs and prehealing expectation with dang-kis' EMs and healing methods. A shared clinical reality is achieved when there are similarities in these areas. Several questions were used for this purpose. For example, during the prehealing interview, patients were asked: "According to you, what caused your problem?" (EMs) and "What are the most important results that you hope to receive from the god?" (Expectation). During the posthealing interview, they were asked: "During the consultation, did the god tell you what your problem was and what caused your problem?" 
Attempts would also be made to elicit dang-kis' EMs from the observation of the healing session.

As Research Question 3 also intended to find out whether perceived helpfulness would be related to patients' understanding of the healing symbols, some questions were asked in the posthealing interview for this purpose. For example, "Do you understand the purpose/contents of the rituals you underwent, or of the recommendations?"

Before the field research, the questionnaire was translated into Chinese, with backtranslation into English to ensure the accuracy of its Chinese version, and was than piloted with three dang-kis' patients.

\section{Data Analysis}

Interview data were first transcribed verbatim by a professional translator. Together with those derived from observation and video recordings of the healing sessions, the data were then analyzed for themes and contents using ATLAS-TI (V5.0; Muhr and Friese 2004), computer-assisted qualitative analysis software. The content analysis was guided by the interpretive and cultural constructivist approach (Gaines 1992; Good 1994; Kleinman 1980) according to the steps recommended by Miles and Huberman (1994). This approach emphasizes the importance of understanding healing as a cultural construct, the intersubjective reality and voices of patients, healers and suffering (Gaines 1992). Compared to the testing of hypotheses and preconceived theories in the positivistic approach, the interpretive approach begins with experienced or perceived forms and moves toward meanings in a cultural context (Peacock 1984).

\section{Results and Discussion}

\section{Healing Symbols of Dang-ki}

Becoming a Dang-ki-Unlike the institutionalized training of religious personnel in organized Buddhism, Taoism or Christianity, there is no formal training for dang-kis. But this does not mean that anyone can become a dang-ki. There are some culturally sanctioned ways through which one is recognized and accepted by the community as a dang-ki (Heinze 1997). Some people are believed to possess the ability to connect to divine forces or to be chosen by the god after being possessed by a deity during festivals while watching shamanistic healing. They are normally encouraged to serve their apprenticeship under an experienced dang-ki. Some become a dang-ki after having some anomalous experiences such as apparitions of deities or being cured by a deity. In the latter case, they are normally asked to serve the god to show their gratitude or to receive ongoing protection against relapse. The "appointment" of a dang-ki can also be hereditary, passing on from parent to child, which is the case of the three dang-kis involved in this study. Whatever the initiation path is, most dang-kis hold a regular job and only serve the role on a part-time basis. Among the three dang-kis in this study, two were full-time practitioners, whereas the third held a full-time job. 
When a person becomes a dang-ki through one of these pathways, he is recognized by the community as the god incarnate-a powerful healing symbol capable of evoking expectancy faith. When devotees believe that the healing power comes from the god rather than the human dang-ki, they may consider the healing complete and effective without questioning (Kirmayer 2004: Littlewood and Dein 1995).

The Healing Context and Process-The three dang-kis involved in this study performed their healing sessions right before the main altars of their respective shrines. Displayed on the altar were statues of Taoist and Buddhist pantheons including the main deities possessing the dang-ki. There were also divine swords, incense burners, incense papers and various religious instruments such as drums, bells and a muyu (木鱼), which is a wooden block used by Buddhists to beat rhythm when chanting scriptures. Facing the altar were a table and a throne that the dang-ki used to conduct his consultation. On the table were a divine seal, a Chinese brush, an ink stick, an ink stone and a stack of $f u$ ( 符; talisman). During the consultation, the dang-ki wrote magical characters and stamped with the divine seal on a fut to activate its power before prescribing it to a patient. One of the dang-kis, who was normally possessed by a benevolent hell god, also had a Chinese abacus on his table for calculating fate.

Before the consultation, the patient took a queue number, took off his or her shoes before entering the shrine hall to greet all the deity statues with joss sticks and then burned the incense papers. The dang-kis' healing sessions went through four stages, beginning with the induction rituals for deity possession, which lasted about 15-30 min. During the induction, the dang-ki yawned, shook his legs, rolled his head or vomited with saliva while his assistants chanted in the background. The types of deities possessing the dang-kis included child gods and hell gods. After being possessed, the dang-ki began the consultation. The length of the consultation ranged from 5 to $30 \mathrm{~min}$, depending on the complexity of the presenting issues. Chinese Hokkien was the main language used during consultation, and no patient faced a language barrier when interacting with the dang-kis. ${ }^{5}$ When there was no more information from the god for the patient, the dang-ki gave a signal that the possessing deity was leaving his body. Finally, he regained consciousness and ended the healing session.

Turner (1979) observed that rituals are often framed in specific space and time with noticeable markers. Inside the ritual is called "sacred," while outside the ritual is called "secular". In the dang-ki shrine as described above, the sacred healing space is demarcated by physical markers, such as the prohibition of footwear in the shrine hall where the consultation was held, by visual markers, such as an altar of religious icons and a table of divine equipment, by olfactory markers, such as incense smell, by auditory markers, such as incantations, and by temporal markers, such as the beginning and ending of the elaborate healing procedures. The solemnity of the sacred space evokes the expectancy faith of the

\footnotetext{
${ }^{5}$ Hokkien is the main Chinese dialect in Singapore. Although previous studies have shown that patients may need a translator to help them make sense of the unintelligible divine language used by their dang-kis (DeBernardi 2006; Kleinman 1980), that is not the case in this study. Even though the use of an unintelligible language may be symbolic proof of the presence of the divine force-dang-ki as a god incarnate - the use of a common language between the healer and the patient may also contribute to a positive healing relationship.
} 
patients when they enter the space, and induces them to surrender their selves to the powerful god. The sacred space and time constitute the total healing symbols and reinforce the efficacy of specific symbolic procedures carried out by the dang-ki during consultation such as his recommendations, prescriptions of $f u$ and herbal treatments.

Since the symbolic structure and meanings of sacred space are consistent with the dang-kis mythic world and EMs, it should be considered part of the mythical world enacted prior to the actual contact between the dang-ki and the patient. It is possible that the patient's improvement may even occur to some extent upon entering the shrine hall (i.e., the sacred space) before his or her personal issues are specifically mapped onto a mythic world by the dang-ki during the consultation.

Presenting Issues-Among the 21 patients taking part in this study, 12 presented physical health issues while 9 presented psychosocial issues (see Table 2). Four of them came to consult on behalf of their relatives (Cases 4, 11, 19 and 22). Dang-kis normally applied more than one healing method (i.e., healing symbols) to treat a particular presenting issue (see Appendix). For example, the dang-ki helped the female patient with hypertension and a history of stroke (Case 1) through a combination of advice, fu, acupuncture and pulse palpation.

Healing Symbols-Because all the three dang-kis employed similar healing methods, their information was combined for analysis. Overall, observations of the 21 healing sessions identified 44 healing methods, which can be classified as follows: (1) words (e.g., advice, recommendations; 43\%); (2) fu (34\%); (3) physical manipulation (e.g., acupuncture, palpation, massage; 14\%) and (4) others (e.g., Chinese herbs, rituals; 9\%). While words and fu were applied for both physical health and psychosocial issues, physical manipulation and herbs were restricted to physical health issues. It is not known whether the acupuncture, massage and herbal medicine prescribed by the dang-kis in this study are based on mainstream traditional Chinese Medicine (TCM) theory practiced by licensed TCM physicians in Singapore, as the dang-kis are not licensed practitioners.

Perceived Helpfulness of the Healing Symbols-As dang-kis applied more than one method to treat a given problem, patients did not necessarily view all methods as being helpful (see Appendix). Eleven patients (52\%) perceived the consultation as helpful and planned to follow all the dang-kis' recommendations. They included 10 regulars (Cases 2, 3, $4,5,6,8,11,13,21$ and 22) and 1 newcomer (Case 15).

Four patients (19\%), including three regulars (Cases 1, 9 and 17) and one newcomer (Case 14), perceived the consultations to be helpful but were unable to follow all recommendations. For example, a female patient (Case 1) who had recovered from stroke admitted to her dang-ki that she had reduced the dosages of her medication from 300 to 50 mg per day without her doctor's permission. The dang-ki advised her to comply with the prescription and gave her other recommendations. When asked whether she would listen to the dang-ki, she answered as follows. ${ }^{6}$

Cult Med Psychiatry. Author manuscript; available in PMC 2016 December 16. 
$P$. I will try my best. But I don't think I could take $300 \mathrm{mg}$ because I'll be very tired. Probably I'll consider $200 \mathrm{mg}$.

$R$ : But didn't shan-cai-tong-zi (the name of the god) advise you to follow the prescribed dosage?

$P$. I worry that I would feel lethargic if I follow the exact dosage. If I'm tired, I would not be able to do other things. Moreover, I don't have to see the doctor any more.

$R$ : That means you don't follow shan-cai-tong-zi's instruction.

$P$. It is not that I want to ignore his instruction. I will try my best to do my part. It is impossible for me to do exactly what he has advised me. He asked me to take a warm foot bath every night. But by the time I come home from work, I'll be too tired to take the bath. I don't have time because it will last at least 20 min. I'll go straight to my bed after a shower. I don't think I could follow his instruction.

Five patients (24\%), who were all newcomers (Cases 10, 12 and 18-20), were not sure whether or not the consultations would be helpful but believed that the effects might not be instant. Nonetheless, they were optimistic and hopeful. Only one patient (5\%; Case 16) considered the dang-ki's advice to be unhelpful.

As a whole, the consultations were considered helpful up to one month of follow-up. Similarly, the Taiwanese dang-ki healing was also considered to be effective based on the patients' self-evaluations (Kleinman 1980; Kleinman and Gale 1982). However, in this study, perceived helpfulness does not mean the total elimination of problems and symptoms. Among the 12 patients presenting health issues, only 1 (Case 3) reported being completely free of symptoms at the one-month follow-up interview. Among the nine patients presenting psychosocial issues, three (Cases 4, 17 and 21) considered their problems to be solved at the follow-up. In total, only $19 \%$ of the patients' problems were completely solved after the consultations. The low remission rate may be due to the nature of presenting issues that were mostly chronic by nature (e.g., hypertension, gastric problems, cancer, muscular atrophy, depression, interpersonal conflicts or unemployment). Our one-month follow-up may be too short for monitoring the progress of the dang-ki treatments.

Nonetheless, a majority of patients continued to consult dang-kis even though personal issues persisted, suggesting that they may adopt a broader view of treatment efficacy and recovery (see Csordas and Lewton 1998; Finkler 2004; Kirmayer 2004; Krippner and Achterberg 2000). This broader view also reflects the approach of dang-ki, which focuses more on the healing of illness rather than the curing of disease (Kleinman and Seeman 2000; Waldram 2000). While curing refers to processes for the removal of pathology, healing refers to the process of restoring subjective, affective, social and spiritual dimensions of illness.

\footnotetext{
${ }^{6}$ In all excerpts, $\mathrm{P}$ refers to patient, $\mathrm{R}$ refers to researcher (the first author), and DK refers to dang-ki. Interviews in Chinese were translated into English.
} 


\section{Explanations for Perceived Helpfulness}

Can perceived helpfulness be explained by a shared clinical reality and transformation of emotions through symbolic manipulations proposed by Levi-Strauss (1963), Dow (1986) and others? Can healing be considered helpful without a "shared" clinical reality, as some patients may not understand the healing process? How do patients define the meanings of helpfulness? To address these questions, we compared patients' EMs and prehealing expectations versus dang-kis' EMs identified during their healing sessions. ${ }^{7}$ Immediately after their consultations and at one-month follow-up, we asked the patients whether they understood the purposes and contents of the dang-kis' advice and treatments, and why they perceived these advice and treatments as helpful. Because regular patients were more likely than newcomers to perceive their consultations as helpful, it is useful to discuss their healing experiences separately.

Regular Patients-Based on their experiences, regular patients can be categorized in four groups.

Group 1: Some patients' EMs and prehealing expectations were consistent with dang-kis' EMs and healing methods. As they spoke the same language-Chinese Hokkien—patients had no problems understanding the purposes and contents of their gods' treatments, advice and recommendations. In Case 3, the patient and dang-ki shared a common cause of his painful arm. The patient felt the consultation to be helpful because the dang-ki's massage (healing symbol) had improved the blood circulation of his jin (筋). ${ }^{8}$ In Case 22, both patient and dang-ki believed that his daughter might have offended negative forces. Thus, the patient found her to be improved after using the futo ward off negative forces. She was able to sit, walk and engage in other motor movements.

Although the symbolic healing model may be able to explain the perceived helpfulness of these patients, what remains unknown is how the common clinical reality has actually developed in these regular patients. Have the patients and dang-kis already shared common EMs right from the first consultation, or has the shared clinical reality only been achieved after a few sessions? As Kleinman (1980) noted, patients tend to change their EMs after consulting health care providers. Thus, future research should focus on new patients and track the convergence of EMs over several sessions.

Group 2: Whether perceived helpfulness is contingent on a shared clinical reality is not straightforward in some cases. In Case 2, the patient's EMs were different from the dangki's EMs. In Cases 5 and 6, the dang-kis were not clear in their EMs. However, this may be because the dang-kis had already stated their EMs in previous consultations. Although some patients (Cases 13 and 21) were unsure of their EMs, their prehealing expectations were met.

\footnotetext{
${ }^{7}$ The dang-kis' EMs were elicited during their consultations. It is impossible to directly interview them for their EMs because they claim to forget their experiences after the spirit possession. It is believed that during the possession the dang-kis soul has left for another realm or his soul remains but is inactive in his body. Since the entity conducting the healing is the god rather than the dang-ki, the dang-ki has no knowledge about the healing process.

8 Jin, a TCM concept, refers to a fibrous cord extending from a muscle (Ou 1988, p. 475).
} 
While most of the patients understood the purposes and contents of their dang-kis' consultations, three female patients (Cases 2, 8 and 13) did not understand some aspects of their consultation. The patient in Case 2 did not understand why she was asked not to eat bread, although she would follow this recommendation. Her gastric problems subsided after her consultation. The patient in Case 8 was unsure of the purpose of the fu prescribed but reasoned that it must be helpful for her condition as her interpersonal relationship at work had improved. The patient in Case 13 did not understand the purpose of the fu given to her, although she felt blessed and protected after eating it. She believed that the fu was helpful but was unable to explain the reason.

Despite the above uncertainties, these patients generally felt the consultation to be helpful. This may be because, first, having a common meaning system or an understanding of the rationale of the specific divine interventions has never been an issue to them. They may be more concerned about the outcome—a pragmatic attitude (Finkler 2004; Lin 1981; Quah 1989). Second, since it is the omnipotent god who provides the consultation, the mere performance of the healing may have already implied its completeness and success (Kirmayer 2004; Littlewood and Dein 1995). Since god and dang-ki are cultural objects conceptualized as powerful and efficient, the healing encounter may lie in the "icon of power and desire" involving surrender of the self to the Other (the god/dang-ki) and the acceptance of the Other as a figure of desire (e.g., compassion) (Lindquist 2007). Thus, the patients should trust the gods and simply follow what they have been told-god is itself a paramount healing symbol. Finally, the perceived helpfulness may be due to nonverbal and paralinguistic aspects of the healing process (Atkinson 1987; Kirmayer 1993). The experiences of these patients suggest that not all patients and dang-kis need to share a common understanding in order for them to interact.

Group 3: For other cases, the interactions among the dang-kis' EMs and the patients' EMs, prehealing expectations, understanding of the healing symbols and perceived helpfulness are a complex process characterized by negotiations, reframing and selective perception.

In Case 1, the female patient's EMs were multidimensional, consisting of physiological (high blood pressure, blockages of arteries), behavioral (irregular meal and imbalanced diet) and psychosocial (work stress) factors. By comparison, her dang-ki's EMs were more narrowly framed in TCM theory: blockages of $j i n$ and $q i$ (cause), using acupuncture to unblock the $q i$ (treatment), avoiding eating carrots and radishes and taking warm foot baths (recommendation). At first glance, the patient and dang-ki did not seem to share a common "clinical reality," yet the patient generally perceived the consultation to be helpful because she felt calmer and more energetic. There are a few explanations. First, her prehealing expectation was rather general-hoping to determine her health condition and how she could get well. Vague expectation can be easily met than more specific expectation. Second, she partially attributed her stroke to the "blockages of arteries," a biomedical concept. In contrast, the dang-ki attributed her stroke to the blockages of jin and $q i$, a TCM concept. For the patient, both concepts were the same since they all referred to "blockages." During the posthealing interview, I (the first author; R) asked her (patient; P) whether the god had told her the cause of her stroke. 
$P$. Yes, it was because of my arteries. I also know that I've problems with my arteries. I've had spurs for a long time, probably at C4 and C5, which have caused my hypertension.

$R$ : Did the god tell you what your problem was?

$P$. He said that my stroke was caused by the arteries. His diagnosis matched with what I've already known.

$R$ : What have you received from the god that you have experienced as helpful, which you do not receive from other people or methods?

$P$. You'll not get such advice and recommendations from other temples. I'm a person who wants to see evidence and consistency. I don't simply listen to what other people tell me. What they tell me has to correspond to what I've already known as I'm aware of my own health condition.

The patient appeared to reframe the dang-ki's TCM diagnoses according to her biomedical EMs and then concluded that the dang-ki's diagnoses were accurate. With this EM match, she became more receptive to the dang-ki's treatments. Thus, she claimed to feel better at her neck after the acupuncture treatment, which was said to clear the "blocked" qi.

The reframing process may also explain the perceived helpfulness of the $f u$ and pulse palpation. One of the causes the patient ascribed for her stroke was work stress and lack of rest. She said: "After having stroke, I easily feel lethargic and lack energy. Now you may find me well but in the next minutes you may find me like a deflating balloon....." Although the dang-ki did not cite work stress as a cause of her stroke, he prescribed fu for calming her mind. In this sense, the $f u$ became therapeutically relevant to her work stress and lack of energy. The patient further added other therapeutic values to the $f u$ even though such values had not been specified by the dang-ki during the consultation.

$R$ : How do you feel after using the fu?

$P$. After using it, my life became very smooth for a few days.

$R$ : What has become smooth?

$P$. Interpersonal relationship. No one quarrels with me and I don't encounter problems at work.

$R$ : How are these changes related to your illness?

$P$. They are all related. If I'm sick, I'll easily lose my temper, and then I'll end up quarreling with my colleagues. After eating the $f u$, I'm able to calm down myself and will not have hot temper.

$R$ : You mean your illness has affected you emotionally. But after taking the $f u$, your emotion has become more stable?

$P$. You're right. But this is mysterious and unexplainable; its effects are invisible.

For the patient, the fu helped her calm her mind, enhance her energy levels and avoid interpersonal conflicts at work. She also thought that the dang-ki's pulse palpation was 
effective in enhancing her energy level, even though this procedure is, strictly speaking, a diagnostic rather than a treatment method in TCM. She said: "On some occasions, I feel a stream of warm energy entering into my body through his pulse palpation. But not everyone has this experience. When he is examining your pulse and you're attentive, you'll experience that warm sensation. For my case, I'll feel very energetic for 3-4 days. He is able to provide you with that kind of energy." In this sense, the pulse palpation is considered helpful because it helps increase her energy level. This interpretation is in line with one of the perceived causes of her stroke-insufficient rest.

Taken together, this patient still finds the dang-ki treatment helpful even though she does not directly share the same system of meaning with her dang-ki. Thus, the dang-ki's treatments include multiple meanings (Turner 1973, 1977) or some components of meaning responses (Moerman 2002; Winkerlman 2000)—whether or not they are the meaning that he intends to evoke. These unintentional meaning responses may also suggest that cultural symbols (e.g., blockages of $j i n$ and $q i$ ) and personal or private symbols (e.g., unresolved interpersonal conflicts, stress) are not separable (Obeyesekere 1990). The cultural symbols may have personal meaning to the patient. By unblocking the jin and $q i$, the interpersonal tension is symbolically unblocked and the energy level is enhanced.

Active reframing also is found in Case 11, where a female patient consulted for her mother's painful arm. Before the consultation, she attributed her mother's symptoms to two possible causes. First, the mother might have contacted evil forces when visiting the ancestral graves. Second, the mother's painful arm might have been due to some nervous disorders in her brain, which could have been caused by her depression as a result of her marital conflicts. The patient's perceived cause of nervous disorder might have been influenced by her colleague who had recently been diagnosed with a nervous disorder.

During the consultation, the dang-ki refuted evil forces as the cause; instead, he offered his TCM explanation. Below is an excerpt from the posthealing interview.

$R$ : During the consultation, did the god mention about the nervous disorders?

$P$. Yes, he did. He said that it is a type of $q i$. To me, it is the nerve. As he talked about jin, he meant the nervous problem, didn't he?

$R$ : Did the god attribute your mother's complaint to a nervous disorder?

$P$. I can't remember. But he said that there was a compression on the jin.

$R$ : Are jin and nerves the same thing?

$P$. They are the same. To my knowledge, both are controlled by the brain.

Similarly to Case 1, the patient reframed the dang-kis' TCM diagnoses according to her biomedical EMs and then concluded that the dang-ki's diagnosis was accurate. The reframing process allows patient and dang-ki to interact without having cognitive dissonance.

These two cases further substantiate the importance of treating symbolic healing as a dynamic process rather than simply a static structure (Kapferer 2004; Turner 1977) in which 
the healer's belief system structures the healing process while the patient remains passive (Dow 1986). One of the possible mechanisms meditating this dynamic process is active reframing.

Group 4: Three patients (Cases 4, 8 and 17) believed that their gods were able to understand them directly in a way similar to telepathic communication. Being powerful gods, they already knew everything about human affairs and were able to read the human mind, and they knew the past, present and future. Therefore, it was not necessary to tell the gods everything during the consultation. For the female patient in Case 4, this "telepathic communication" suited her character because she was poor at expressing feelings and thoughts. The god could read her thoughts directly without misinterpretation: unlike mortals, who often distorted and misunderstood each others' words. She came to consult the god for her son's interpersonal problems at work. She was not sure about the cause and hoped to find out whether the god had already started to help her and how he had helped her so far. Below is an excerpt from her consultation.

$D K$ : You always worry.

$P$. You know I am worried about something?

$D K$ : If you are not worried, you won't look so upset. Do you understand? You won't be able to solve problems when you're upset. The most important thing is that you have to find out the cause and then solve it. If you keep on thinking negatively, you won't be happy. Do you understand me? The more anxious you are, the more distracted you will be. Because you're anxious, that is why you came to consult the god. You're afraid of failure, and you're afraid of not being able to complete your work. As the saying goes: "One becomes more alert when there are serious matters while one becomes confused when there are small matters." This is the human problem.

$P$. Are you saying that I've become confused again?

$D K$ : If you're not distracted easily, you'll not be so careless. Do you understand me? Sometimes, you've to be forgiving. Otherwise, you will create troubles for yourself. So don't blame others. You want to help others because you're afraid that they are jobless. But when the problem occurs, you shouldn't blame others. Because you're already a grownup, you must know what is right and what is wrong. But the other party also has to understand. Now you're here, I've already answered your query by expelling your poison. But if you prefer to continuously poison yourself, I will not stop you either. I'm helping people to release their poison. However, I don't understand why people like to poison themselves. They are deeply troubled because they often think negatively. And so their lives become so meaningless. Don't take in other people's poison. You should learn to reflect so as to solve your problems. If people are good, so are you. Do you understand? Don't be angry when others don't appreciate your kindness. You're willing to help other people when they need help. The more upset you are, the more nervous you will be. Do you get my points? 
$P$. What I want to tell you today is that...

$D K$ : I've already answered your questions. So you don't have to tell me. You know better than I do.

After the consultation, I (the first author) asked her:

$R$ : Do you understand the purpose of his advice just now?

$P$. Of course, I do. However, as being a human being, it is difficult to take things easy. Although he has guided me and asked me not to think too much, I'll ruminate whenever having problems. That's why he said that he has to help detoxify me, yet I continue taking poison. In other words, he advised me not to think too much. Preempt problem before it happens, solve the problem when it happens, and face the reality and don't split hairs. The god will help me to solve every problem. However, as being a human, we'll become unhappy when facing problems.

$R$ : Do you understand what he (the god) has told you?

$P$. I do. This is because we are spiritually connected. I understand everything he told me. Whatever thing that you don't understand, I can understand.

$R$ : Did you tell him directly your problems?

$P$. No, I didn't. It would be a long story if I want to tell him.... But he has already advised me what to do.

$R$ : Is today's consultation helpful to you, and why?

$P$. It's helpful. Before coming here I was very unhappy. But now, after talking to him, I've become relaxed. I know that the problem was not as serious as I thought. I also know how to manage it.

In the follow-up interview one month later, I (the first author) asked her whether the divine advice had been helpful. She answered: "Yes. He said that when the right time comes, everything will be clear. So when I received the message from my son yesterday, I was not that surprised. The problem is solved. Now that my son can look for a new business partner...."

As shown, the conversation between the dang-ki and the patient is vague, the presenting problems are unclear and the dang-ki's recommendations are nonspecific. The vague advice is open to interpretation and may result in self-fulfilling prophecy. Basically, he advises the patient not to think too much but to think positively. He advises her to maintain good interpersonal relationships, not to blame others and to learn to reflect on herself. Interestingly, the advice on maintaining good interpersonal relationships is more relevant to her son than to her because it is her son who faces this problem. Yet the dang-ki seems to treat her as the one having this problem. She may have used this information to make sense of her son's interpersonal problems.

The ability for the god to know things that humans cannot possibly know is also observed in Cases 8 and 17. For example, the depressed patient who presented interpersonal problems in Case 8 found the divine advice to be more helpful and practical than her psychiatrist's help. 
She said: "The medicine can only put my condition under control. Although it can help me to be less anxious, I've to deal with so many things in life, such as my work." She also thought that counseling could console her but would not be able to solve her work problems. In contrast, the god could tell her what she had not been aware of so that she would know how to manage the interpersonal situation at work. She said:

The god is different; he can help you solve the problems. The psychiatrist can only help "balance your heart," and help you ventilate so that you feel better. But when you talk to him about your work, ask him why the colleagues are not happy, and in what ways they are not happy, he doesn't have the answers. He would only advise you not to be bothered by them. But the god is different. As a god, he has already known why they were unhappy with me. So after listening to him, I have a clearer idea of the situation, and therefore I am able to solve the problems.

In sum, the "god" or the dang-ki demonstrates his power by his ability to know unrevealed events. This ability further invokes the patients' faith in the healer, which promotes their perceived helpfulness of the healing. Thus, the patients' faith is in part dependent on whether the god has "correctly guessed" their stories and predicaments. For example, the god might have correctly pointed out the problem of the patient's son in Case 4. However, even if the dang-ki does not have the correct answers, the patient may simply ignore the wrong answers, and only listen to and remember the correct ones. This cognitive process may be termed "selective perceived helpfulness." Similarly to reframing, selective perceived helpfulness may help patient and dang-ki to construct a common experiential reality by focusing on relevant components but filtering out inconsistencies during their interaction.

Regular Attendance and Shared Clinical Reality-Although the 13 regular patients generally regarded the dang-ki consultations as being helpful, their perceived helpfulness may be partially due to their regular attendance, which enables them to continuously receive their dang-kis' support. Likewise, Finkler (1981) found that Mexican patients' perceived recovery from their ailments was associated with the length of contact with their shamans. As long as they continued to maintain contact with their shamans, they tended to ignore or minimize their condition. As such, shamanism can be considered an everyday healing activity instead of a sudden "miraculous" healing (Csordas 1994). Its efficacy may be incremental and cumulative, rather than instantaneous, or may be induced through a series of "booster" sessions and constant re-exposure to the healing effects.

According to the shrine assistants, about $60 \%$ of the patients are regulars. People are prone to return to the same healer after receiving positive healing experiences. Such experiences in turn reinforce the perceived helpfulness of their subsequent consultations. For example, the patient having chronic depression (Case 8) had attended consultation on a regular basis for six to seven years. She explained: "This is because I always learn something after listening to him. He wants me to come here every week. By doing so, I will gradually improve and learn to take things easy." Another patient (Case 21) believed that her current interpersonal problem would be resolved because her dang-ki had cured her skin problems before.

Through regular attendance, patients are more susceptible to dang-kis' influence; as such, their EMs are likely to converge over time. Thus, a shared clinical reality observed in any 
particular healing session may be the accumulative effects of positive healing experiences and perceived helpfulness derived from previous healing sessions. Although the symbolic healing model proposes that the success of any healing system depends on the healer's ability to enact a shared mythic world and then to emotionally transform the patient's experiences by attaching her emotions to the transactional symbols, this process may evolve over several sessions, whereby the effects are cumulative rather than instantaneous. To see how this process actually evolves, future research should focus more on new clients, starting from the first consultation and following them through several sessions.

Newcomers-After discussing the healing experiences of the regulars, we now move onto the newcomers. Among the eight newcomers, two (Cases 14 and 15) perceived their consultations to be helpful and planned to follow most of the dang-kis' recommendations, five (Cases 10, 12 and 18-20) were unsure whether their consultations would be helpful, but believed the effects were not instant and one (Case 16) considered his consultation unhelpful.

Group 1: For Cases 14 and 15 (see Appendix), the patients and their dang-kis shared some aspects of EMs, particularly those concerning TCM health beliefs. As the English-speaking patient in Case 15 provided the most detailed account of his emotional and cognitive changes, his experiences are discussed in detail here.

He consulted for his digestive problems, which he attributed to overeating and exposure to poisonous gas while working at a waste treatment plant. He wanted his health back, his digestive system to recover, to have an appetite and to get stamina to work. Although the god's diagnosis was pretty brief and simple- "eating too much"-it partially matched the patient's perceived causes (overeating). The divine treatments also were simple, asking the patient to avoid cold food and cold drink, which might worsen his health because his body was "warm." This advice was based on the "hot/cold" theory in TCM (Beinfield and Korngold 1991). Next, the god conducted a ritual by using a Chinese brush to paint a small red dot on the space between his two eyebrows for protective purposes. The god also prescribed two types of $f u$ as part of the treatments. As the patient was afraid of having cancer, he reported feeling peaceful, with a sense of security, after using the $f u$.

$P$. After drinking the talismans, I am more at peace and I don't think of the sickness so much and can concentrate on other things. [They] give me some sort of security.

$R$ : This is what he [the dang-ki] told you?

$P$. Yes.

$R$ : How is your digestive condition after drinking the talismans?

$P$. Since I visited the shrine two months ago, I've noticed some improvements in my digestive system. I started taking in food but on-off still....

$R$ : How did you take the talisman?

$P$. There are two talismans-one is for drinking, another is for cleansing the body. Both must be combined. 
$R$ : What is the purpose of the cleansing?

$P$ Cleansing is to remove all the evil or bad things in you. Drinking is to give you a peace of mind throughout the night.

$R$ : Do you think the treatments, talismans and recommendations are helpful?

$P$. In a way. My sixth-sense tells me that, after drinking, I feel more peaceful. I have a better rest as compared to the past. Then, my mind doesn't run wild. Previously, my mind ran wild, thinking all sorts of nonsensical things. Now after taking the talismans, I feel more peaceful during my sleep every night. I can sleep very well.

$R$ : What do you mean by "your mind is running wild"? Any examples?

$P$. Like, "my sickness is getting worse, and I end up dying. Then, I go to hell." I have all these weird dreams seeing coffins, and people dying on the street. Their symptoms are always similar to mine.

As a whole, the patient felt energetic, secure, and peaceful after visiting the shrine.

$R$ : What have you received from the god that you have experienced as helpful which you don't receive from other people?

$P$. I feel my mind more relaxed as compared to seeing a Western GP. After consulting the god, it gives me a sense of security. I noticed something funny. Before stepping into this temple, I had no stamina. But when I stepped out of the house, on the way to the temple, my stamina seemed to improve.

$R$ : After seeing the god?

$P$. No, before seeing the god, on the way to the temple. Everything seemed to be fine. There were a few occasions, I did not feel well, for example, like just now [my] stomach didn't feel well and got gases, I didn't feel like coming but I forced myself to come. But when I stepped out of the house [his home], everything just went off. Now, I can talk. It is funny to me. But when I sit here, I don't think of my illness at all. But when I am at home, my mind runs wild.

$R$ : But how did you explain all these?

$P$. I can't explain. I feel something funny. That's why I am more sincere to praying to this god now. I take it more seriously. I've noticed a few occasions. Not only once or twice but so many times my sister asked whether I could walk, I said I could, I just changed and less than a few minutes everything seemed normal. When I reached the temple, everything is ok. Seemingly, my sickness has all gone. I just relax my mind.

$R$ : So how do you feel now?

$P$. I feel very relaxed like a normal person.

To some extent, the dang-ki treatments have met his expectations, particularly by improving his appetite and his energy level through the "effects" of talismans. Emotionally, he also felt more relaxed and peaceful. From the symbolic healing perspective, the dang-ki evoked a 
mythic world framed in TCM and supernatural beliefs and then attached the patient's emotions and needs (e.g., fear of death and contracting cancer, regaining his appetite and stamina) to the transactional symbols (i.e., the blessing red dot and fu). Subsequently, the dang-ki manipulates the symbols to transform the patient's experience.

Group 2: Among the five patients (Cases 10, 12 and 18-20), although most of their EMs and prehealing expectations were congruent with their dang-kis' EMs and healing symbols, they were unsure whether the consultations would be helpful because they had yet to see any concrete results. But they reasoned that the dang-kis' healing might take a while before showing any effects. The patient from Case 10 told me: "Until now, I still don't have any confidence [in the treatment] because this is only the second time I saw him [the god], it is impossible to expect any instant results." He believed that his skin problems would be healed gradually but not so soon. Nonetheless, he felt more energetic and calm after using the $f u$, which, according to the god, could detoxify his body.

The female patient with floaters in her eyes (Case 12) said: "I don't know how he could help me. Only when the results appear slowly would you know whether he has helped you...." She was unsure whether the fu prescribed would help her eyes, thinking that it might be meant for her worry.

In Case 18, the god advised the patient to be a bookmaker. He would not succeed if he chose to work in a mainstream job because of his karma. That explained why he had been unemployed and his career path had not been smooth. However, the patient was rather skeptical of this recommendation, as he was very reluctant to work in this kind of job. $\mathrm{He}$ needed some time to consider the god's recommendation. Similarly, the patients in Cases 19 and 20 were also uncertain about the outcomes of their consultations, although they would carry out the god's instructions. Of course, they hoped for the best.

As a whole, these patients did not report being completely free of symptoms or issues after one month. Nor had they returned for a second consultation. However, it is premature to treat these cases as failures. Our follow-up may be too short to detect any substantial changes over time, as the presenting issues may be chronic by nature. These patients may return for a second consultation, or more, after our one-month follow-up.

A practical question arises from these cases: At what point in the healing should we evaluate whether dang-ki is helpful, and whether the patients have recovered and restored their functioning? As Waldram (2000) noted, the timeframe for determining cure in biomedicine may be more definite because of its use of precise technology (e.g., lab tests) to determine the onset and course of disease. By contrast, as shamanism focuses on psychosocial and spiritual transformations rather than simply physical functioning, the timeframe is less precise in shamanism, which may involve a lifelong process of seeking healing without a clearly defined end point. Instead of being a sudden "miraculous" healing, dang-ki may be a partial and everyday healing method. Its effectiveness may be incremental (see Csordas 1994), with ever-changing criteria and definitions of efficacy (see Waldram 2000). 
Hence, this timeframe issue should be considered when applying the symbolic healing framework to understand shamanistic efficacy. As mentioned before, the enactment of a common clinical reality or the therapeutic manipulations of healing symbols may be a more complex and dynamic process, evolving over a couple of interactions between patient and healer, rather than a simple process that can be achieved within a single session.

Group 3: Of the 21 patients interviewed in this study, only 1 considered his consultation unhelpful. This case is interesting because it is a new case. The patient did not have any prior contact with the dang-ki before his consultation. We were able to follow the patient's healing experience from the first consultation to a follow-up eight months later.

The male patient wanted to ask the god why his life had not been stable and smooth, particularly his career, as he had been unemployed for three years. For him, his career problem might have been due to bad luck, power struggle at work and evil spirits. He first encountered a strange phenomenon when working in an old food and beverage outlet where he heard some spirits calling his name. I (the first author) asked him if this paranormal experience was connected to his career problem. He answered in English: "I guess so because there is no other reason that can explain why my career has not been stable for three years. You tend to have this kind of encounter in unlucky years ... or it may be because I've offended the spirit...." So he hoped to find out from the god whether it was the spirit or other unknown factors that had caused his career problem.

During the consultation, the dang-ki read the patient's palm and told him that he was supposed to lead a prosperous life because he was born on the same day as the Jade Emperor, the King of the Taoist gods. However, his good fortune was somehow "broken," and his "career line" was short. The god believed that the real cause was an abortion his exwife had induced ten years ago. As abortion is a form of murder, his present problem was his retribution (i.e., karma). Although the patient was amazed by the god's ability to know about the abortion that he had not disclosed to anyone, he disagreed with the god.

$P$. I don't know whether she lied to me. Yes, she told me about the abortion. But I don't know how true it is. I don't even know whether she had been pregnant. If she were pregnant, I would definitely know whether she had an abortion or not.

$D K$ : Although you don't know, it doesn't mean that it's not true, right? So there is a possibility, right?

$P$. So, what can I do now?

$D K$ : You can only save yourself now.... You're supposed to have a good fate. But because something happened in your life, your fate has been changed. You have to do charity work to cultivate merits so as to mend the fate and get back to a better life.

$P$. But ... my Lord, I've not done anything wrong.

$D K$ : Have you aborted your baby?

$P$. I don't know. But I don't think this has happened. I don't know whether she had told me the truth. 
$D K$ : But why are you so sure that she had lied to you?

$P$. If there was an abortion, she should have told me!

$D K$ : She had already told you! Yet, you don't believe her. So what do you want her to do?

$P$. To be frank, I don't believe her.

$D K$ : That means you don't think you have a bad fortune.

$P$. I do think my life is "bad." But I've not done anything wrong.

$D K$ : Your wife has told you but you don't believe her. Now, I'm telling you the same thing but you don't believe me either. Since you don't believe me, why did you come to see me? Do you understand me? [At this juncture, the god obviously loses his patience. He raises his voice to show his anger.]

$P$. Yes.

$D K$ : So to mend your fate, you've got to cultivate gong-de [ 功德; merits, good deeds].

$P$. But one needs a lot of money to cultivate gong-de.

$D K$ : Are you telling me that because you don't have money, you can't cultivate gong-de? So, are you trying to tell me that the main reason you came here is to get rich so that you can cultivate gong-de?

$P$. Of course not. I did not imply that.

$D K$ : Then why did you tell me that you're poor? What I want you to know is that you don't have to be rich before you can cultivate gong-de.... For example, giving a donation to build a temple is not gong-de. We donate money to build a temple because we want to thank the god and the saint. This is not gong-de. Gong-de means helping in old folks' homes and orphanages. Look at me, I'm also poor, but I've been helping so many people over the past ten years.

About 10 min later, the patient finally accepted the abortion as the cause of his career problem. During the posthealing interview, he told me that although the god had helped him to understand the cause of his turbulent life, he was disappointed and upset:

He [the god] did not help me. He did not help me. Directly, he did not. Indirectly, yes, he has advised me. Indirectly, he has advised me to help myself by cultivating good deeds. This is why I protested to him. When doing good deeds, I need a lot of money. I told him this ... but he said no, no, no.... I said that I've led a decent life without hurting and harming people. I don't steal.... He said that these were not good deeds.... When I came here, I wanted to settle certain things. I wish to know exactly what has actually happened. And this objective has been achieved.... However, I feel disappointed because nobody can help me, only I can help myself... If there were some spiritual beings following me, at least I could do something, or he can do something. He can advise me to do something about it by 
getting rid of the spirit. But to him, this is all because of what I did twenty years ago!

Apparently, the patient would prefer the spirit to be the cause of his career problem so that it could be easily handled by the god. Shamans are generally perceived to be better than other practitioners (e.g., biomedical or TCM physicians) at curing issues caused by supernatural entities (Ahern 1975; Kleinman and Sung 1979; Quah 1989). However, instead of spirits, the dang-ki attributed his problem to his ex-wife's abortion and his wrongdoing. Although the patient claimed to accept this causal attribution toward the end of the consultation and during the interview immediately after the session, he did not seem to be completely convinced. At a follow-up interview conducted 8 months later, I asked him whether he still agreed with this cause. He answered: "I'm not sure. I'm not sure whether this is the cause." $\mathrm{He}$ also asserted that one must have a lot of money to cultivate good deeds. Thus, not surprisingly, he did not follow the god's recommendation. He did not experience any changes following the consultation; his luck had not been improved, and he was still unemployed.

From the symbolic healing perspective, the dang-ki failed to enact a common mythical world to help the patient. Even though they do share a general supernatural framework, they do not agree on a particular causal attribution. The patient may still favor spirit to abortion as the cause of his problem. More specifically, they patient and dang-ki may disagree on the locus of responsibility and control (Sue and Sue 2008). Locus of responsibility refers to causal attribution of events, whereas locus of control refers to one's ability to influence an event outcome. For the dang-ki, the patient's "bad life" was caused by retribution (karma) as a result of the sinful abortion. In order to reverse his life, he has to cultivate merits through personal efforts. As such, the god adopts an external locus of responsibility and an internal locus of control. Although the patient may also adopt an external locus of responsibility (abortion or spirit as the cause), he feels powerless to control his fate and prefers an active divine intervention - an external locus of control. Overall, this case illustrates how the healer attempted to enact a mythical world to convince and persuade the patient how his life could be transformed. The patient reacted initially with acceptance of the cultural explanation but later with rejection of its validity.

From another viewpoint, the patient's apparition of spirits may be interpreted as an unconscious projection of intrapsychic conflicts or a personal symbol (Obey-esekere 1990). The unsuccessful transformation may be attributed to the dang-ki's failure to apply a cultural symbol that was personally meaningful to the patient. A personal symbol has both motive (archaic motivation of childhood) and meaning (reflexivity). However, the meaning of the patient's personal symbol appears to be shallow, in the sense that there is an overdetermination of motive but an underdetermination of meaning. An underdetermination of meaning will not help transcend the domination of motive. The dang-ki may attempt to make use of pre-existing cultural symbols (karma, cultivating good deeds through charity work) to edit the meaning of his personal symbols—moving from spiritual disturbance to sinful abortion—so that he could "progress" (see Obeyesekere 1990). From the Buddhist and Taoist perspective, such progression can be achieved by cultivating good karma by 
becoming involved in charity work. But the overdetermination of the deep motivation may prevent the patient from integrating his personal symbol with this cultural symbol.

Findings from Case 16 suggest that instead of merely asking whether dang-ki or shamanism is helpful, it is also important to ask whether they are unhelpful. Although the notion of efficacy is culturally defined to ensure success, shamanism must also have failures just like any other healing systems (Finkler 2004; Kleinman 1980; Nguyen 2008; Schieffelin 1996). Based on her work with dang-kis in Malaysia, DeBernardi (2006) observed that their healing outcomes range from beneficial to ineffective or inappropriate. The tendency for patients to visit shrine after shrine for their unresolved problems suggests that hope does not work in all cases. However, DeBernardi did not explain how failures had been defined and measured. "In order to properly understand efficacy, it is essential to both comprehend what constitutes a lack of efficacy and examine specific instances of failure alongside those of success" (Waldram 2000, p. 610). Future research should focus more on cases of failure in shamanistic healing so as to arrive at a more complete picture of its healing dynamics.

\section{Summary and Conclusion}

In this study, we have explored the perceived helpfulness and therapeutic process of dang-ki from the symbolic healing perspective. We were interested in finding out the healing symbols employed in dang-ki, whether patients find these healing symbols helpful and whether their perceived helpfulness can be explained by a shared experiential reality and their understanding of the purpose and contents of the healing symbols. Below are the main findings.

\section{Meanings of Helpfulness}

In line with other researchers' observations (Csordas and Lewton 1998; Finkler 2004; Kleinman and Sung 1979; Kirmayer 2004; Krippner and Achterberg 2000), the meanings of helpfulness varied across patients. They perceived the consultation to be helpful after experiencing improvements or changes in their physical (e.g., gastric relief, better blood circulation, more energetic), cognitive-affective (e.g., calmness, peacefulness, sense of security, self-awareness, hopefulness), interpersonal (e.g., avoidance of interpersonal conflict) or spiritual (e.g., the presence of divine forces, communicating with god) functioning.

\section{Multiple Healing Symbols and Differential Perceived Helpfulness}

Dang-kis employed more than one healing method or symbol to treat a presenting problem. These symbols were mostly words (e.g., advice, recommendations), followed by fu, physical manipulation (e.g., acupuncture, pulse palpation, massage) and others such as Chinese herbs and rituals. Half of the patients viewed all methods to be helpful and intended to follow all recommendations, while the other half were either unable to follow all recommendations or not sure whether the consultations would be helpful. Only one patient perceived his consultation to be unhelpful. Hence, although dang-ki was generally perceived as helpful, it is useful to examine patients' perception and evaluation of specific healing processes and methods. As patients play active rather than passive roles during the healing process, they do 
not necessarily consider all treatment methods to be meaningful and useful. They actively interpret, reframe and select recommendations and treatments relevant to their needs.

\section{Symbolic Healing is a Deep, Complex, Interactional and Ongoing Process}

Although to some extent the symbolic healing model is useful for explaining the perceived helpfulness of dang-ki, the symbolic healing process is far more complex and dynamic. While a relationship between perceived helpfulness and shared clinical reality is quite clear in some cases, it is less straightforward in others. Among the latter cases, some patients and dang-kis do not share all aspects of their EMs. Nonetheless, they manage to work together in those aspects they agree on. Other patients may start off with EMs different from those of their dang-kis, but their EMs converge through reframing and reinterpretation. Although some patients are not clear in their EMs, or do not understand the rationales of certain aspects of their consultation, in general, they consider their healing sessions to be helpful.

It is important to note that most of the patients who perceive their consultation to be helpful are regular patients. A common worldview may be established through regular attendance, whereby patients receive continuous support from the dang-kis. The fact that these regulars continuously return for consultation may imply the dang-kis' ability to meet their needs and induce hope in them.

Taken together, several impressions emerge from these cases. First, for some patients, healing efficacy does not necessarily depend on a common meaning system. Other contributing factors may be equally important, for example, expectations, faith, hope and pragmatic attitude. Second, some patients are active participants in the healing process and jointly construct a common clinical reality through reframing and selective perception. This finding is contrary to Dow's (1986) view that in symbolic healing it is the healer's belief system that structures the healing process while the patient remains passive. Third, perceived helpfulness and shared clinical reality are likely to be accumulative effects carried over several healing sessions. Positive healing experiences evoke faith and hope, which in turn induce positive healing experiences in subsequent sessions. Fourth, the dang-ki's treatments may include some components of meaning responses, whether or not they are the meaning that he intends to evoke. Last but not least, the patient's perceived helpfulness and the dang$k i$ 's success in enacting a common mythic world may occur to some degree when the patient enters the sacred space in the shrine before their actual healing contact. Thus, the sacred space should be considered part of the mythic world within a symbolic healing framework.

\section{Limitations and Future Research}

Although dang-ki is generally perceived as helpful, this perception is mainly derived from regular patients rather than newcomers. As there were more regulars than newcomers (13 vs. 8 ) in this study, this finding may be skewed. There are two possible reasons why regulars outnumber newcomers. First, patients who find dang-ki helpful may be more likely than patients who do not find it helpful to continue treatment at the shrines. Failed cases do not normally return (Kleinman 1980). Next, regulars may be more willing than newcomers to participate in research interviews. They may be more eager and enthusiastic to share their positive healing experiences with the researcher and to pass on the legacy of their gods. 
Thus, future research should involve more new cases, beginning with their first healing session and continuing to monitor their perceived helpfulness over several sessions or longer. Such a focus will also help to show how a common mythic world is constructed over time. A major limitation of this study is that it followed the patients over only a one-month period.

As this study involves only three dang-kis and 21 patients, its findings may not be generalizable to other shrines and patients. More shrines, dang-kis and patients should be included in future studies. We also hope that our research protocol, with its focus on therapeutic process and perceived helpfulness, can be applied to studying shamanistic healings in other cultures to allow comparative studies across different shamanistic systems.

\section{Acknowledgments}

We are deeply grateful to the following people (in alphabetical order) for their help during the fieldwork: C. W. Chan, Margaret Chan, Aaron Choo, Timothy Pwee, Jave Wu, Victor Yue and members of the Singapore Chinese Heritage Group. We also owe a debt of thanks to all participants (dang-kis, helpers, patients and attendees), whose personal stories and healing experiences have enlightened and touched us. Without them, this study would not have been possible. Finally, we thank the anonymous reviewers for their invaluable suggestions.

\section{References}

Ackerman SE. Divine Contracts: Chinese New Religious and Shamanic Movements in Contemporary Malaysia. Journal of Contemporary Religious. 2001; 16(3):293-311.

Ahern, EM. Sacred and Secular Medicine in a Taiwan Village: A Study of Cosmological Disorders. In: Kleinman, A.Kunstadter, P.Alexander, ER., Gale, JL., editors. Medicine in Chinese Cultures: Comparative Studies of Health Care in Chinese and Other Societies. Bethesda, MD: National Institutes of Health; 1975. p. 91-113.

Atkinson JM. The Effectiveness of Shamans in an Indonesian Ritual. American Anthropologist. 1987; 89(2):342-355.

Balzer, MM. Shamanism. In: Levinson, D., Ember, M., editors. Encyclopedia of Cultural Anthropology. Vol. 4. New York: Henry Holt; 1996. p. 1182-1186.

Baruss, I. Alterations of Consciousness: An Empirical Analysis for Social Scientists. Washington, DC: American Psychological Association; 2003.

Beinfield, H., Korngold, E. Between Heaven and Earth: A Guide to Chinese Medicine. New York: Ballantine Books; 1991.

Castillo, RJ. Lessons from Folk Healing Practices. In: Tseng, WS., Streltzer, J., editors. Culture and Psychotherapy: A Guide to Clinical Practice. Washington, DC: American Psychiatric Association Press; 2001. p. 81-101.

Chan, M. Tang-ki: Chinese Spirit Medium Worship. Singapore: SNP; 2006. Ritual Is Theater, Theater Is Ritual.

Chong SA, Mythily, Lum A, Chan YH, McGorry P. Determinants of Duration of Untreated Psychosis and the Pathway to Care in Singapore. International Journal of Social Psychiatry. 2005; 51(1):5562. [PubMed: 15864975]

Costantino G, Malgady RG, Rogler LH. Cuento Therapy: A Culturally Sensitive Modality for Puerto Rican Children. Journal of Consulting and Clinical Psychology. 1986; 54(5):639-645. [PubMed: 3771881]

Csordas, TJ. The Sacred Self: A Cultural Phenomenology of Charismatic Healing. Berkeley: University of California Press; 1994.

Csordas TJ, Lewton E. Practice, Performance, and Experience in Ritual Healing. Transcultural Psychiatry. 1998; 35(4):435-512.

D’Anglure, BS. Shamanism. In: Barnard, A., Spencer, J., editors. Encyclopedia of Social and Cultural Anthropology. London: Routledge; 1997. p. 504-508. 
DeBernardi, J. The Way That Lives in the Heart: Chinese Popular Religion and Spirit Mediums in Penang, Malaysia. Palo Alto, CA: Stanford University Press; 2006.

Desjarlais RR. Healing through Images: The Magical Flight and Healing Geography of Nepali Shamans. Ethos. 1989; 17(3):289-307.

Dow J. Universal Aspects of Symbolic Healing: A Theoretical Synthesis. American Anthropologist. 1986; 88(1):56-69.

Elliott, AFA. Chinese Spirit-Medium Cults in Singapore. London: Athlone Press; 1955.

Fidler, RC. Spirit Possession as Exculpation, with Examples from the Sarawak Chinese. In: Winzeler, RL., editor. The Seen and the Unseen: Shamanism, Mediumship and Possession in Borneo. Borneo Research Council Monograph Series. Vol. 2. Phillips, ME: Borneo Research Council; 1993. p. 207-232.

Finkler K. Non-medical Treatments and Their Outcomes. Culture, Medicine and Psychiatry. 1980; 4:271-310.

Finkler K. Non-medical Treatments and Their Outcomes, Part Two: Focus on Adherents of Spiritualism. Culture, Medicine and Psychiatry. 1981; 5:65-103.

Finkler, K. Traditional Healers in Mexico: The Effectiveness of Spiritual Practices. In: Gielen, UP.Fish, JM., Draguns, JG., editors. Handbook of Culture, Therapy, and Healing. Hillsdale, NJ: Lawrence Erlbaum Associates; 2004. p. 161-174.

Frank, JD., Frank, JB. Persuasion and Healing: A Comparative Study of Psychotherapy. 3. Baltimore: John Hopkins University; 1991.

Freeman P. A Festival for the Dead in Japan. Transcultural Psychiatry. 1998; 35(4):551-555.

Gaines, AD., editor. Ethnopsychiatry: The Cultural Construction of Professional and Folk Psychiatries. Albany: State University of New York Press; 1992.

Good, BJ. Medicine, Rationality, and Experience: An Anthropological Perspective. Cambridge, UK: Cambridge University Press; 1994.

Groleau D, Young A, Kirmayer LJ. The McGill Illness Narrative Interview (MINI): An Interview Schedule to Elicit Meanings and Modes of Reasoning Related to Illness Experience. Transcultural Psychiatry. 2006; 43(4):671-691. [PubMed: 17166953]

Heinze, RI. Trance and Healing in Southeast Asia Today. Thailand: White Lotus Press; 1997.

Helms, JE., Cook, DA. Using Race and Culture in Counseling and Psychotherapy: Theory and Process. Boston: Allyn and Bacon; 1999.

Holbrook B. Chinese Psychosocial Medicine, Doctor and Dang-ki: An Inter-cultural Analysis. Bulletin of the Institute of Ethnology, Academia, Sinica. 1975; 37:85-111.

Jakobsen, MD. Shamanism: Traditional and Contemporary Approaches to the Mastery of Spirits and Healing. New York: Berghahn Books; 1999.

Kapferer B. Ritual Dynamics and Virtual Practice: Beyond Representation and Meaning. Social Analysis. 2004; 48(2):35-54.

Kirmayer LJ. Healing and the Invention of Metaphor: The Effectiveness of Symbols Revisited. Culture, Medicine and Psychiatry. 1993; 17:161-195.

Kirmayer LJ. Asklepian Dreams: The Ethos of the Wounded-Healer in the Clinical Encounter. Transcultural Psychiatry. 2003; 40(2):248-277. [PubMed: 12940648]

Kirmayer LJ. The Cultural Diversity of Healing: Meaning, Metaphor and Mechanism. British Medical Bulletin. 2004; 69:33-48. [PubMed: 15226195]

Kleinman, A. Patients and Healers in the Context of Culture. Berkeley: University of California Press; 1980.

Kleinman A, Gale JL. Patients Treated by Physicians and Folk Healers: A Comparative Outcome Study in Taiwan. Culture, Medicine and Psychiatry. 1982; 6:405-423.

Kleinman, A., Seeman, D. Personal Experience of Illness. In: Albrecht, GL.Fitzpatrick, R., Scrimshaw, SC., editors. Handbook of Social Studies in Health and Medicine. London: Sage; 2000. p. 230-242.

Kleinman A, Sung LH. Why Do Indigenous Practitioners Successfully Heal? Social Science and Medicine. 1979; 13B:7-26. 
Kreisman JJ. The Curandero's Apprentice: A Therapeutic Integration of Folk and Medical Healing. American Journal of Psychiatry. 1975; 132(1):81-83. [PubMed: 1088843]

Krippner SC. Conflicting Perspectives on Shamans and Shamanism: Points and Counterpoints. American Psychologist. 2002; 57(11):962-977. [PubMed: 12564209]

Krippner, SC., Achterberg, J. Anomalous Healing Experiences. In: Cardena, E.Lynn, SJ., Krippner, S., editors. Varieties of Anomalous Experience: Examining the Scientific Evidence. Washington DC: American Psychological Association; 2000. p. 353-396.

Laird J. Sorcerers, Shamans, and Social Workers: The Use of Ritual in Social Work Practice. Social Work. 1984 Mar-Apr;:123-129.

Laufer B. Origin of the Word Shaman. American Anthropologist. 1917; 19(3):361-371.

Lee BO. Chinese Indigenous Psychotherapies in Singapore. Counseling and Psychotherapy Research. 2002; 2(10):2-10.

Lee, BO. Clients as Co-healers. In: Lui, HWE., Wong, SS., editors. Reflections on Counseling: Developing Practice in Schools. Singapore: Pearson Prentice Hall; 2006. p. 56-74.

Lee RLM. Continuity and Change in Chinese Spirit Mediumship in Urban Malaysia. Bijdragen tot de Taal, Land-en Volkenkunde. 1986; 142(23):198-214.

Levi-Strauss, C. Structural Anthropology. New York: Basic Books; 1963.

Li, FY. Shou-jing: A Magico-medical Phenomenon. In: Li, ZT., editor. Taoist Studies and the Religious Culture of China. Taiwan: Zhong-Hua; 2003. p. 280-328.

Lin, KM. Traditional Chinese Medicine Beliefs and Their Relevance for Mental Illness and Psychiatry. In: Kleinman, A., Tseng, YL., editors. Normal and Abnormal Behavior in Chinese Culture. Dordrecht: D. Reidel; 1981. p. 25-111.

Lindquist G. Beyond Meaning: Globalization and the Icons of Power. Anthropology and Medicine. 2007; 14(3):307-320. [PubMed: 27268745]

Littlewood R, Dein S. The Effectiveness of Words: Religion and Healing among the Lubravitch of Stamford Hill. Culture, Medicine and Psychiatry. 1995; 19:339-383.

Miles, MB., Huberman, AM. Qualitative Data Analysis: An Expanded Sourcebook. 2. London: Sage; 1994.

Moerman, DE. Explanatory Mechanisms for Placebo Effects: Cultural Influences and the Meaning Response. In: Guess, HA.Kleinman, A.Kusek, JW., Engel, LW., editors. The Science of the Placebo: Towards An Interdisciplinary Research Agenda. London: BMJ Books; 2002. p. 77-107.

Muhr, T., Friese, S. User's Manual for ATLAS.ti 50. 2. Berlin: Scientific Software Development; 2004.

Nguyen TH. Yin Illness: Its Diagnosis and Healing within Len Dong (Spirit Possession) Rituals of the Viet. Asian Ethnology. 2008; 67(2):305-321.

Obeyesekere G. Symbolic Foods: Pregnancy Cravings and the Envious Female. International Journal of Psychology. 1985; 20:637-662.

Obeyesekere, G. The Work of Culture: Symbolic Transformation in Psychoanalysis and Anthropology. Chicago: University of Chicago Press; 1990.

Ou, M. Chinese-English Dictionary of Traditional Chinese Medicine. Hong Kong: Intellectual; 1988.

Parks FM. The Role of African American Folk Beliefs in the Modern Therapeutic Process. Clinical Psychology: Science and Practice. 2003; 10(4):456-457.

Peacock J. Symbolic and Psychological Anthropology: The Case of Pentecostal Faith Healing. Ethos. 1984; 12(1):37-53.

Quah, SR. The Triumph of Practicality: Tradition and Modernity in Health Care Utilization in Selected Asian Countries. Singapore: Institute of Southeast Asian Studies; 1989.

Schieffelin, E. On Failure and Performance: Throwing the Medium Out of the Séance. In: Laderman, C., Roseman, M., editors. The Performance of Healing. New York: Routledge; 1996. p. 59-90.

Sherzer, J. Kuna Ways of Speaking: An Ethnographic Perspective. Austin: University of Texas; 1983.

Sue, DW., Sue, D. Counseling the Culturally Diverse: Theory and Practice. 5. New York: John Wiley and Sons; 2008.

Tseng, WS. Traditional and Modern Psychiatric Care in Taiwan. In: Kleinman, A.Kunstadter, P.Alexander, ER., Gate, JL., editors. Culture and Healing in Asian Societies: Anthropological, Psychiatric and Public Health Studies. Cambridge: Schenkman; 1978. p. 311-328. 
Turner V. Symbols in African Ritual. Science. 1973; 179(4078):1100-1105. [PubMed: 17788268]

Turner V. Process, System, and Symbols: A New Anthropological Synthesis. Daedalus, Discoveries and Interpretations: Studies in Contemporary Scholarship. 1977; 1(3):61-80.

Turner V. Frame, Flow and Reflection: Ritual and Drama as Public Liminality. Japanese Journal of Religious Studies. 1979; 6/4:465-499.

Vitebsky, P. Shamanism. Oklahoma: University of Oklahoma Press; 2001.

Walach H, Jonas WB. Placebo Research: The Evidence Based for Harnessing Self-Healing Capacities. Journal of Alternative and Complementary Medicine. 2004; 10(1):S-103-S-112. [PubMed: 15630827]

Waldram JB. The Efficacy of Traditional Medicine: Current Theoretical and Methodological Issues. Medical Anthropology Quarterly. 2000; 14(4):603-625. [PubMed: 11224983]

Welch JS. Ritual in Western Medicine and Its Role in Placebo Healing. Journal of Religion and Health. 2003; 42(1):21-33.

Winkerlman, M. Shamanism: The Neural Ecology of Consciousness and Healing. London: Bergin \& Garvey; 2000.

Yeh CJ, Hunter CD, Madan-Bahel A, Chiang L, Arora AK. Indigenous and Interdependent Perspectives of Healing: Implications for Counseling and Research. Journal of Counseling and Development. 2004; 82(4):410-419.

Young A. Some Implications of Medical Beliefs and Practices for Social Anthropology. American Anthropologist. 1976; 78(1):5-24.

Zhang, YQ. Unpublished Master's Dissertation. Qinhua University; 1996. An Anthropological Study of the Holy Medicine Prescribed by Dang-ki in Taiwan.

\section{Appendix}

See Appendix Table 3. 


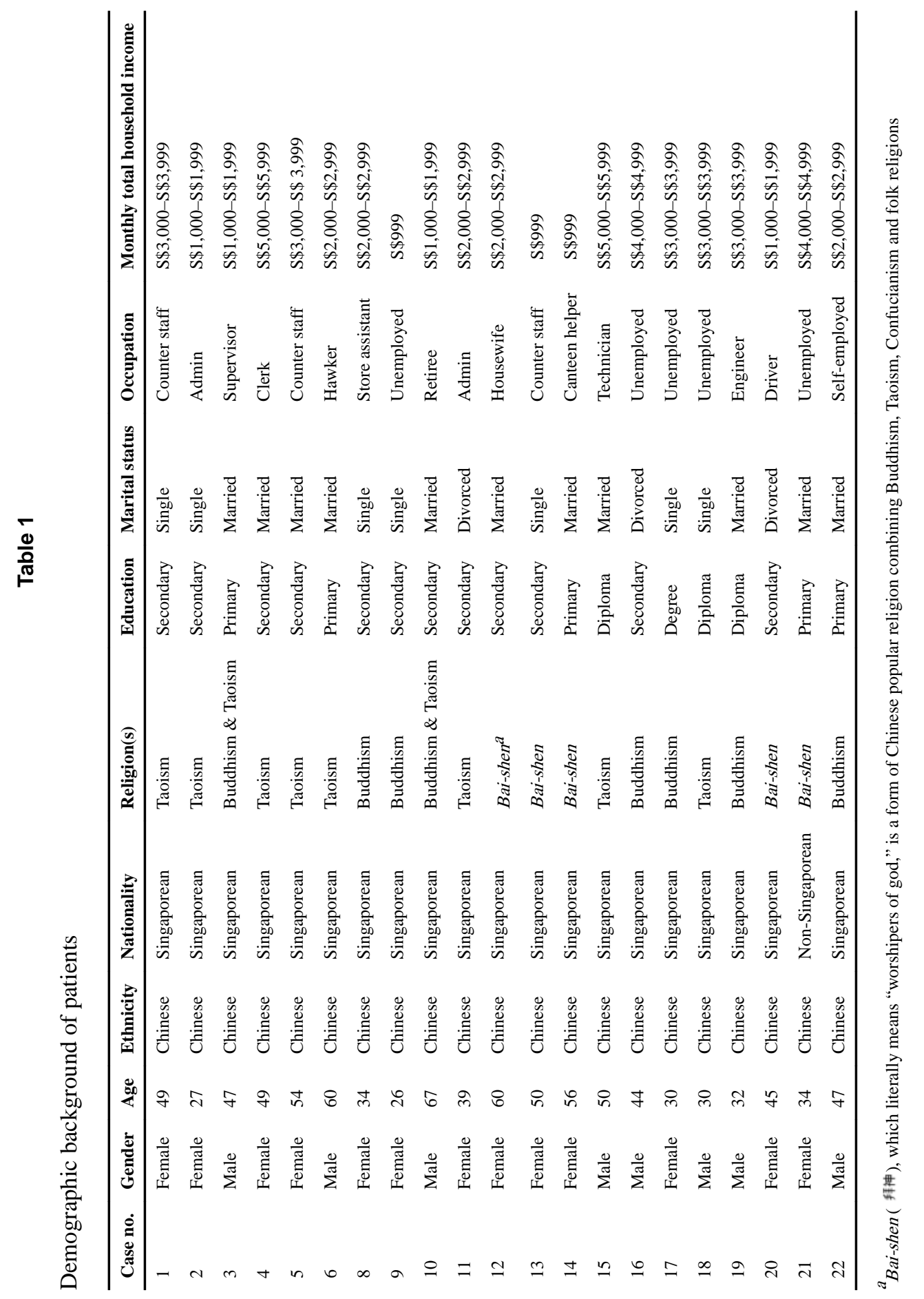




\section{Table 2}

Specific issues presented to dang-kis by patients

\begin{tabular}{|c|c|c|c|c|}
\hline Case no. & Main issue & Issue belonged to & Type of patient ${ }^{a}$ & $\begin{array}{l}\text { No. of times } \\
\text { consulting for this } \\
\text { issue }\end{array}$ \\
\hline \multicolumn{5}{|c|}{ Physical health issue $(N=12)$} \\
\hline 1 & Hypertension \& had stroke 1 yr ago & Self & Regular & Many \\
\hline 2 & Gastric problems: frequent belching \& poor appetite for 2 to $3 \mathrm{yr}$ & Self & Regular & Many \\
\hline 3 & Pain in both arms for about 2 mo & Self & Regular & First \\
\hline 6 & $\begin{array}{l}\text { Diagnosed with lymphoma } 1 \mathrm{yr} \text { ago } \& \text { is in remission. Came here } \\
\text { for blessing }\end{array}$ & Self & Regular & Many \\
\hline 9 & Suffering from muscular atrophy \& blurred vision for $8 \mathrm{mo}$ & Self & Regular & Many \\
\hline 10 & $\begin{array}{l}\text { Skin problem for } 2 \text { mo: feeling itchy \& self- conscious about the } \\
\text { scars }\end{array}$ & Self & Newcomer & Second \\
\hline 11 & Pain in the right arm for about 4 days & Mother & Regular & First \\
\hline 12 & Floaters in the eyes & Self & Newcomer & First \\
\hline 13 & $\begin{array}{l}\text { Gastric problems for about } 6 \text { mo: indigestion, feeling bloated after } \\
\text { meals, \& weight loss }\end{array}$ & Self & Regular & Many \\
\hline 14 & $\begin{array}{l}\text { Having frequent flu-like symptoms (running \& stuffy nose) for } 2 \\
\text { wk }\end{array}$ & Self & Newcomer & Second \\
\hline 15 & Gastric problem: indigestion \& weight loss for about 8 mo & Self & Newcomer & Third \\
\hline 22 & Daughter ill for a few years & Daughter & Regular & Many \\
\hline \multicolumn{5}{|c|}{ Psychosocial issues $(N=9)$} \\
\hline 4 & Being anxious about her child's work problems & Son & Regular & First \\
\hline 5 & Problems with colleagues & Self & Regular & Many \\
\hline 8 & $\begin{array}{l}\text { Depression for about } 20 \text { yr: problems with colleagues \& problems } \\
\text { in completing work tasks }\end{array}$ & Self & Regular & Many \\
\hline 16 & Unemployment \& life has not been smooth for $3 \mathrm{yr}$ & Self & Newcomer & First \\
\hline 17 & Unemployment \& career has not been smooth for $3 \mathrm{yr}$ & Self & Regular & Many \\
\hline 18 & Bad luck \& unemployment for about $3 \mathrm{yr}$ & Self & Newcomer & Third \\
\hline 19 & $\begin{array}{l}\text { Having frequent nightmares for } 4 \text { mo \& wondering whether it is } \\
\text { due to evil forces }\end{array}$ & Wife & Newcomer & First \\
\hline 20 & Financial difficulty & Self & Newcomer & Second \\
\hline 21 & Problems with ex-colleagues for many months & Self & Regular & First \\
\hline
\end{tabular}

${ }^{a}$ Regular, patients having attended healing sessions for more than 1 year; newcomer, one to three visits 


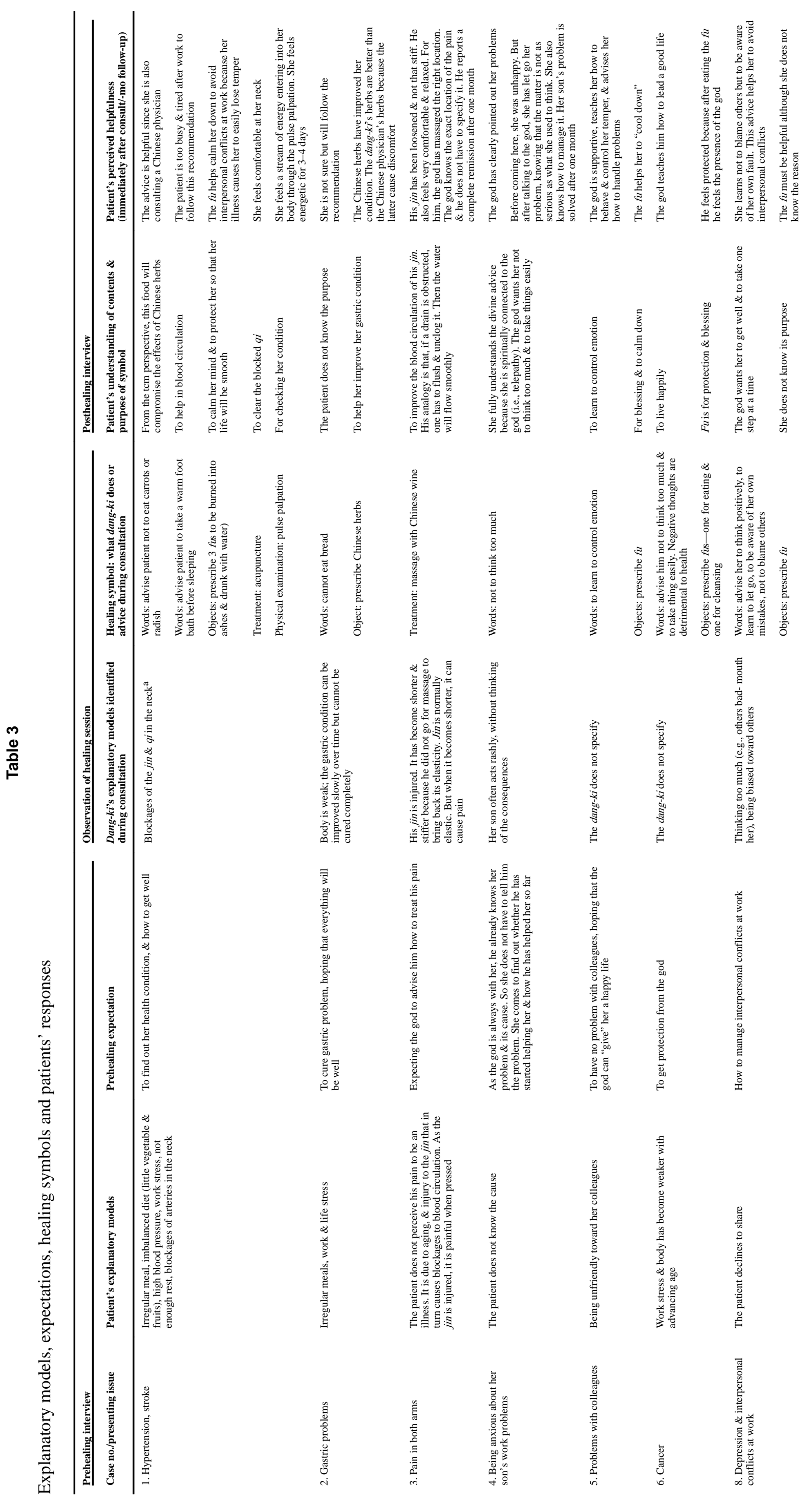




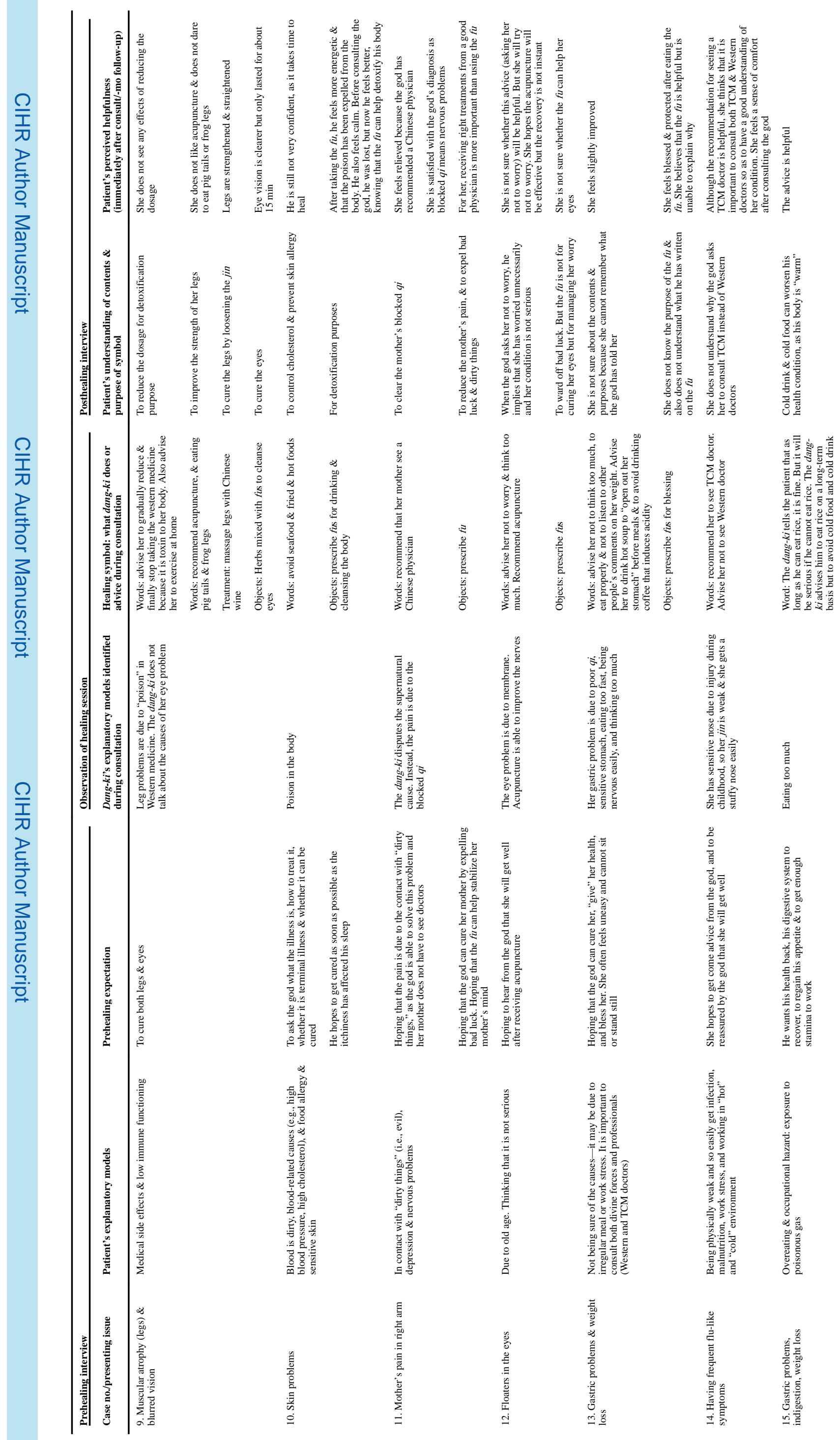



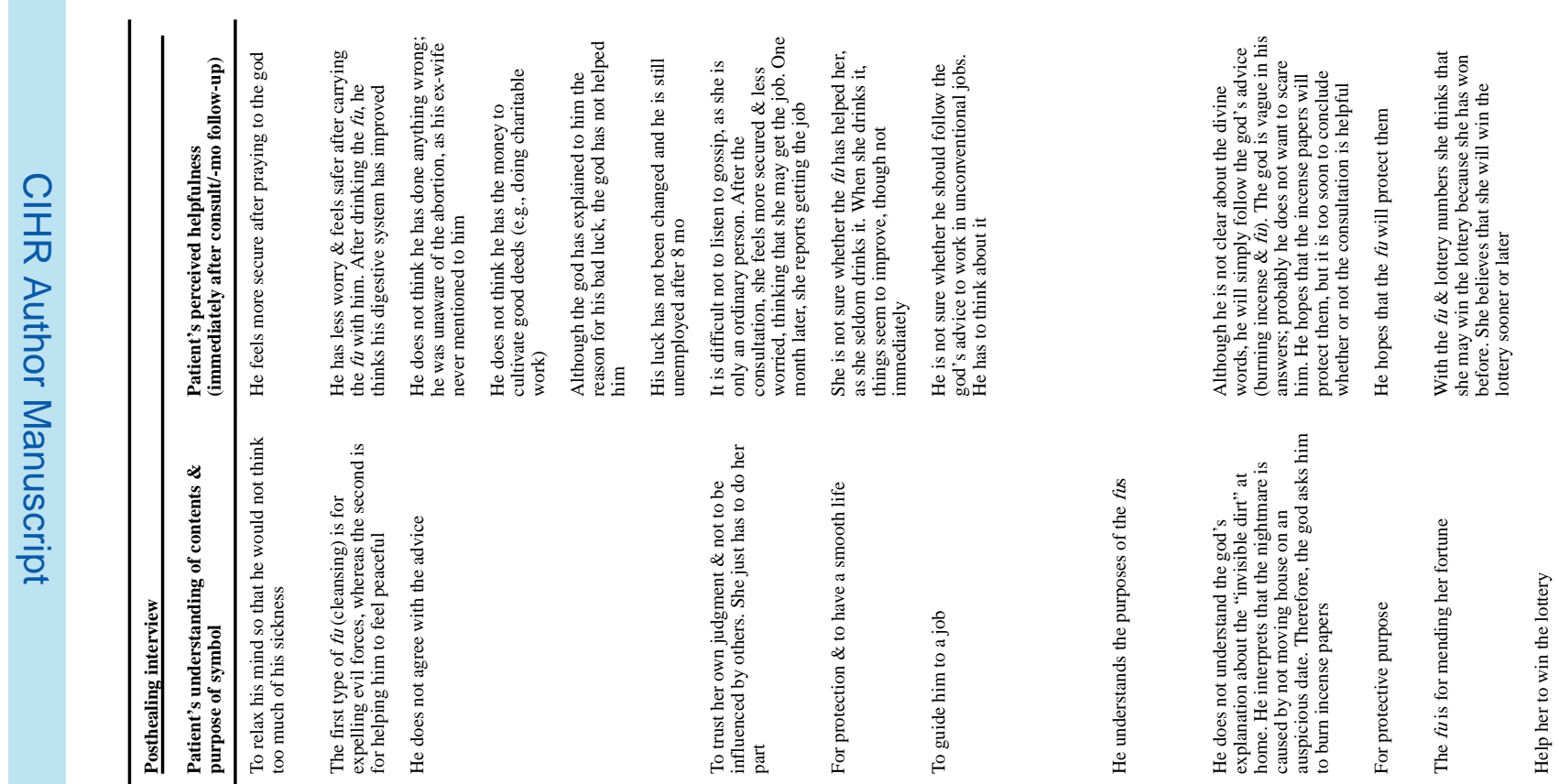

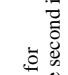

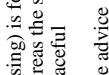

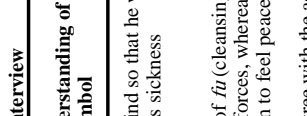

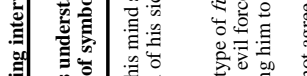

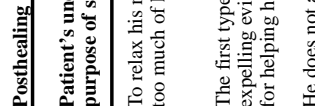
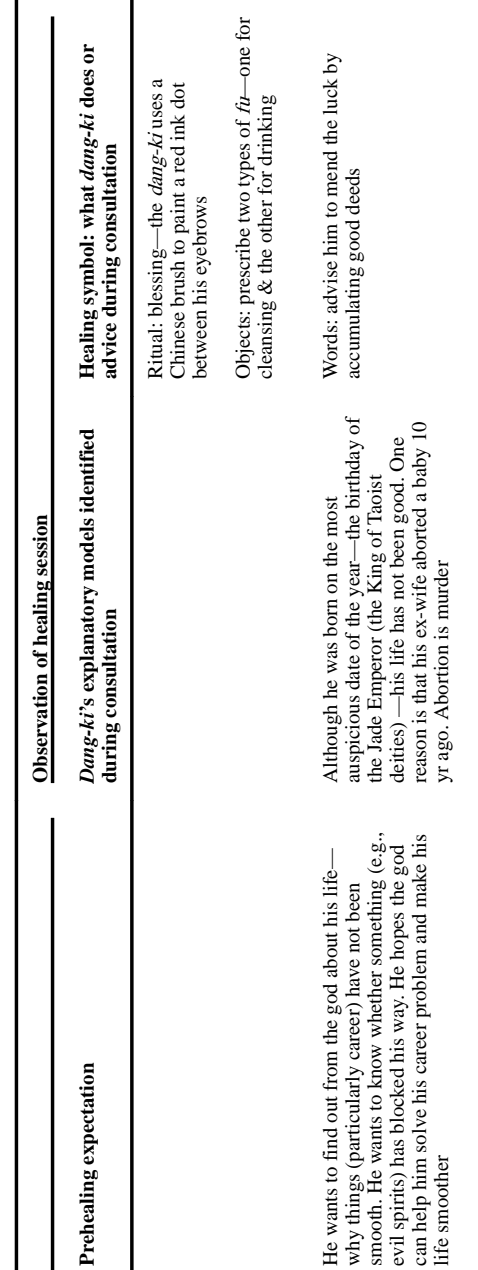

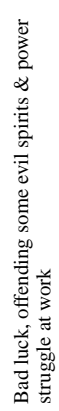

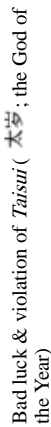

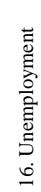
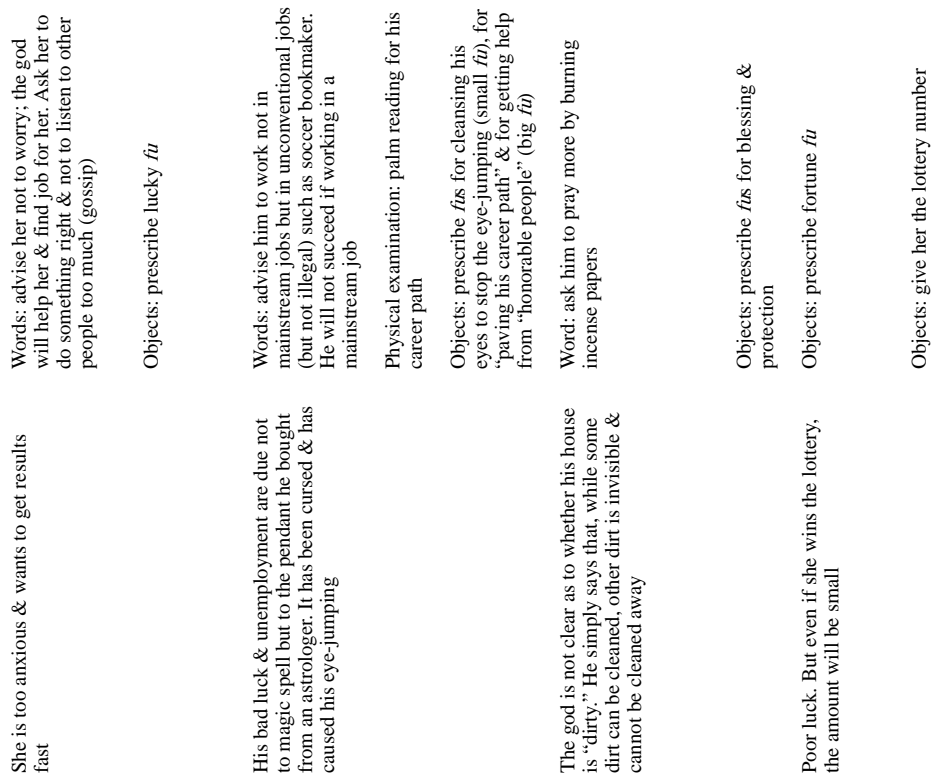

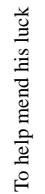

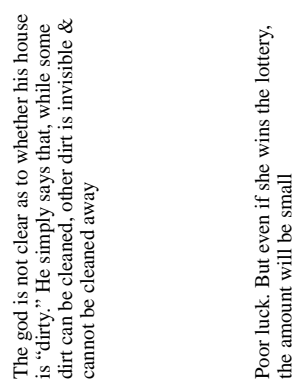

递
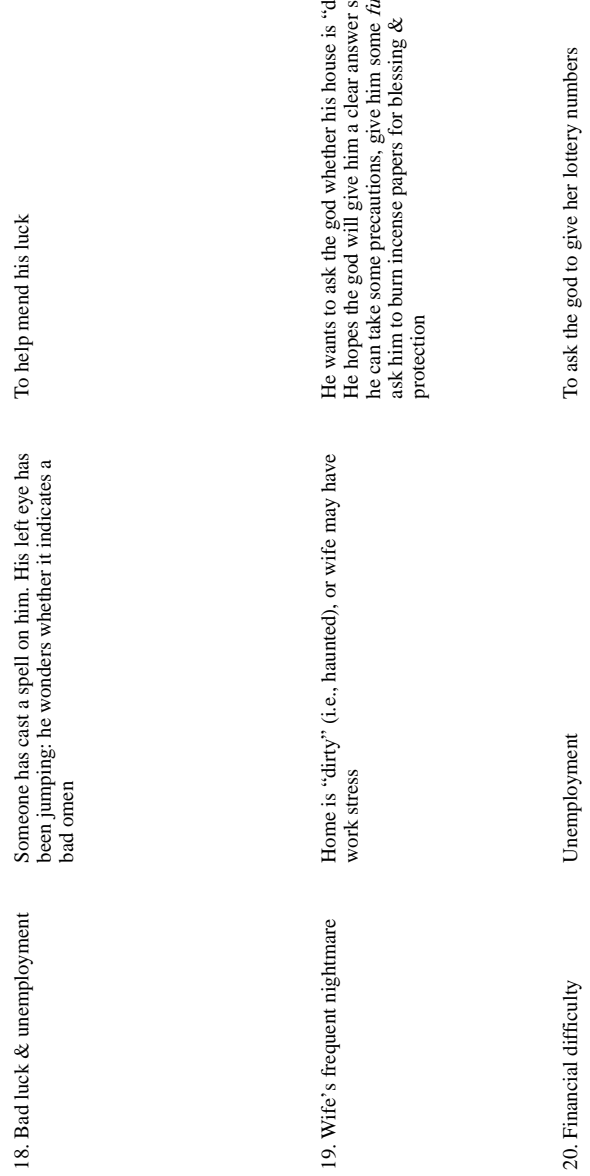


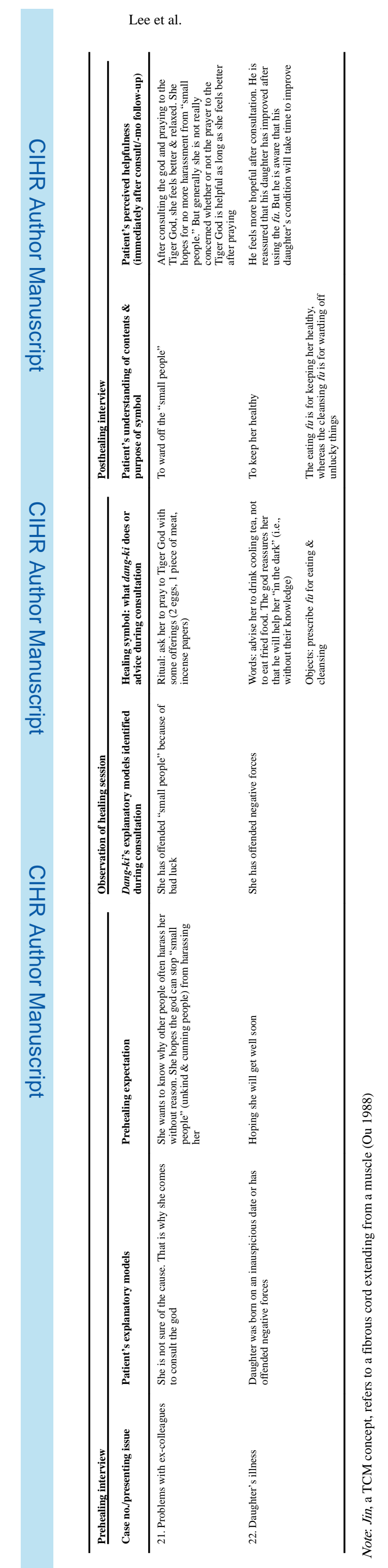

Page 37 